\title{
Comparison of Amino Acid PET to Advanced and Emerging MRI Techniques for Neurooncology Imaging: A Systematic Review of the Recent Studies
}

\author{
Brittany M. Stopa $\mathbb{D}^{1,2}$ Csaba Juhász $\mathbb{D}^{1,3,4}$ and Sandeep Mittal $\mathbb{D}^{1,2,5}$ \\ ${ }^{1}$ Virginia Tech Carilion School of Medicine, Roanoke, VA, USA \\ ${ }^{2}$ Fralin Biomedical Research Institute, Roanoke, VA, USA \\ ${ }^{3}$ Departments of Pediatrics, Neurology, Neurosurgery, Wayne State University School of Medicine, Detroit, MI, USA \\ ${ }^{4}$ PET Center and Translational Imaging Laboratory, Children's Hospital of Michigan, The Karmanos Cancer Institute, Detroit, \\ MI, USA \\ ${ }^{5}$ Carilion Clinic Neurosurgery, Roanoke, VA, USA
}

Correspondence should be addressed to Sandeep Mittal; sandeepmittal@vt.edu

Received 18 September 2020; Revised 23 October 2020; Accepted 17 November 2020; Published 20 January 2021

Academic Editor: Neil Vasdev

Copyright (c) 2021 Brittany M. Stopa et al. This is an open access article distributed under the Creative Commons Attribution License, which permits unrestricted use, distribution, and reproduction in any medium, provided the original work is properly cited.

\begin{abstract}
Introduction. Standard neuroimaging protocols for brain tumors have well-known limitations. The clinical use of additional modalities including amino acid PET (aaPET) and advanced MRI (aMRI) techniques (including DWI, PWI, and MRS) is emerging in response to the need for more accurate detection of brain tumors. In this systematic review of the past 2 years of the literature, we discuss the most recent studies that directly compare or combine aaPET and aMRI for brain tumor imaging. Methods. A PubMed search was conducted for human studies incorporating both aaPET and aMRI and published between July 2018 and August 2020. Results. A total of 22 studies were found in the study period. Recent studies of aaPET with DWI showed a superiority of MET, FET, FDOPA, and AMT PET for detecting tumor, predicting recurrence, diagnosing progression, and predicting survival. Combining modalities further improved performance. Comparisons of aaPET with PWI showed mixed results about spatial correlation. However, both modalities were able to detect high-grade tumors, identify tumor recurrence, differentiate recurrence from treatment effects, and predict survival. aaPET performed better on these measures than PWI, but when combined, they had the strongest results. Studies of aaPET with MRS demonstrated that both modalities have diagnostic potential but MET PET and FDOPA PET performed better than MRS. MRS suffered from some data quality issues that limited analysis in two studies, and, in one study that combined modalities, overall performance actually decreased. Four recent studies compared aaPET with emerging MRI approaches (such as CEST imaging, MR fingerprinting, and SISTINA), but the initial results remain inconclusive. Conclusions. aaPET outperformed the aMRI imaging techniques in most recent studies. DWI and PWI added meaningful complementary data, and the combination of aaPET with aMRI yielded the best results in most studies.
\end{abstract}

\section{Introduction}

The standard-of-care neuroimaging modality for detection of brain tumors is contrast-enhanced magnetic resonance imaging (CE-MRI), which is a critical component of the clinical management strategy, from diagnosis to prognosis as well as treatment response assessment [1]. However, standard MRI has some important clinical limitations [2]. It can- not be relied upon for definitive diagnosis or tumor grading; therefore, pathology remains the diagnostic benchmark. Conventional MRI has limited utility for predicting prognosis or outcomes, and it has limited correlations with tumor genetic profile data, which is increasingly central to tumor prognostication [3]. Likewise, the application of standard MRI is limited for response assessment and treatment monitoring, because it cannot distinguish between treatment 
effects (including radiation necrosis) and tumor progression with high accuracy. Given these limitations, there is clearly a need for more sophisticated advanced neuroimaging modalities that can augment and resolve the gaps in the existing standard imaging. The advanced noninvasive neuroimaging modalities whose clinical use is emerging in response to this need include amino acid positron emission tomography (aaPET) and advanced MRI (aMRI). Especially as hybrid PET/MRI imaging systems become available in more clinical settings [4], the opportunities to use these two important modalities within the same exam are likely to facilitate profound advances in neurooncologic imaging capabilities.

PET is a noninvasive imaging modality that involves administration of a radioactive (positron-emitting) tracer and subsequent photon detection by a PET scanner to quantify radioactivity accumulation in tissue. Depending on which tissue accumulates the tracer or its metabolites, the PET scan will reveal a differential profile of radioactivity. aaPET tracers provide information pertaining to the tissue, depending on the transport and metabolism of that specific amino acid molecule. aaPET imaging has been endorsed by the Response Assessment in Neuro-Oncology Working Group and the European Association for Neuro-Oncology for diagnosing and grading tumors, delineating extent for resection or radiation planning, monitoring treatment, and diagnosing progression in gliomas and certain metastatic brain tumors [5-7]. The main radiotracers for aaPET in neurooncology include L-[methyl]- ${ }^{11} \mathrm{C}$-methionine (MET), ${ }^{18}$ F-fluoroethyl-tyrosine (FET), ${ }^{18}$ F-fluoro-L-dihydroxy-phenylalanine (FDOPA), and ${ }^{11} \mathrm{C}$-alpha-methyl-L-tryptophan (AMT). MET was the first aaPET radiotracer developed and widely used [8]; however, the $20 \mathrm{~min}$ half-life of the carbon-11 isotope limits its widespread clinical utility. FET was developed based on tyrosine transport from blood to tumor tissues, and the use of fluorine-18 with 110 min halflife in this tracer makes FET much more amenable for routine clinical use and distribution $[9,10]$. FET has been widely adopted and is approved for clinical neurooncology use in several European countries. FDOPA was a tracer originally developed for imaging the basal ganglia $[11,12]$, as it measures dopamine synthesis, but it has recently been adopted for neurooncologic imaging, as it utilizes the same L-type amino acid transporter system as other aaPET tracers [13]. AMT was originally developed for imaging serotonin synthesis [14], and it was later adopted for imaging epileptic tissue [15] and CNS neoplasms [16, 17]. A unique aspect of AMT PET is its potential to image the activity of the immunosuppressive kynurenine pathway $[13,18]$. There are additional aaPET radiotracers under development and investigation, but we will limit our scope in the present review to these four main tracers while focusing on the most recent developments.

The leading aMRI techniques for clinical neurooncology imaging are diffusion-weighted imaging (DWI), perfusionweighted imaging (PWI), and magnetic resonance spectroscopy (MRS). DWI creates tissue contrast reflective of the random, microscopic translational motion of water molecules in the body. The diffusivity is measured by apparent diffusion coefficient (ADC). In tumors, free diffusivity of water mole- cules can be impaired by high cell density and/or high nuclear-to-cytoplasmic ratio; thus, reduced diffusivity is a surrogate marker for increased tumor cellularity [19]. DWI can improve detection, characterize the biology of tumors, and monitor treatment response; it is also being investigated for tumor grading [20]. PWI is used to noninvasively measure cerebral perfusion, through hemodynamic measurements like cerebral blood volume, blood flow, and mean transit time [21]. The PWI techniques of dynamic susceptibility contrast (DSC) and arterial spin labeling (ASL) operate on similar physical principles, with tagged blood causing a transient, localized change of the MR relaxation rates depending on perfusion properties. However, DSC uses an exogenous contrast material and ASL uses arterial water as an endogenous tracer. PWI has been mainly used in stroke assessment but also found a useful clinical application for identifying and grading brain tumors [22], such as with cerebral blood volume maps to assess the neovascularity of tumors, which may correlate with tumor grade and malignant histology [21]. MRS is used to create a nuclear magnetic resonance spectrum of the chemical content of tissue, which allows for noninvasive assessment of the molecular composition of tumors [23]. MRS may thus be used alongside standard MRI to improve brain tumor diagnostic accuracy and assessment of treatment changes [23]. With MRS, there is a specific molecular signature (the spectrum) related to both the interaction of the magnetic field with each molecule's unique distribution of electrons and the interactions between nuclei. The areas of peaks within the spectrum are associated with (but not a direct measure of) concentration. This allows for noninvasive assessment of molecular composition of tumors, including several important metabolites. As tumor grade increases, the metabolites $\mathrm{N}$-acetylaspartate and creatine decrease, while the metabolites choline, lipids, and lactate increase, resulting in changes to the ratios between peak areas [24].

As these two distinct advanced noninvasive neuroimaging modalities have evolved in recent years, there has been a growing interest in better characterizing the unique strengths of each modality, how aMRI and aaPET techniques compare, and how they might be used in a complementary manner to elucidate a more complete understanding of a brain tumor's profile. The recent review article by Lohmann et al. [25], focusing on studies completed prior to 2018, established a basis for understanding these imaging modalities in a neurooncologic context. In four studies that combined MRS with aaPET, one with MET PET [26] and three with FET PET [27-29], a high diagnostic accuracy of the individual and combined modalities was reported, but with inconsistent spatial congruence [25]. In 13 comparative studies of PWI with aaPET, seven used FET PET with DSC PWI [30-36], four used MET PET with DSC PWI [26, 37-39], one used FDOPA PET with DSC PWI [40], and one used FDOPA PET with ASL [41]. Overall, aaPET was superior to PWI for diagnosing recurrent glioma, and PWI did not accurately represent the extent of glioma, although there was some variability between studies using different methodology about whether the modalities resulted in spatially congruent images. The seven studies of DWI combined with aaPET, 


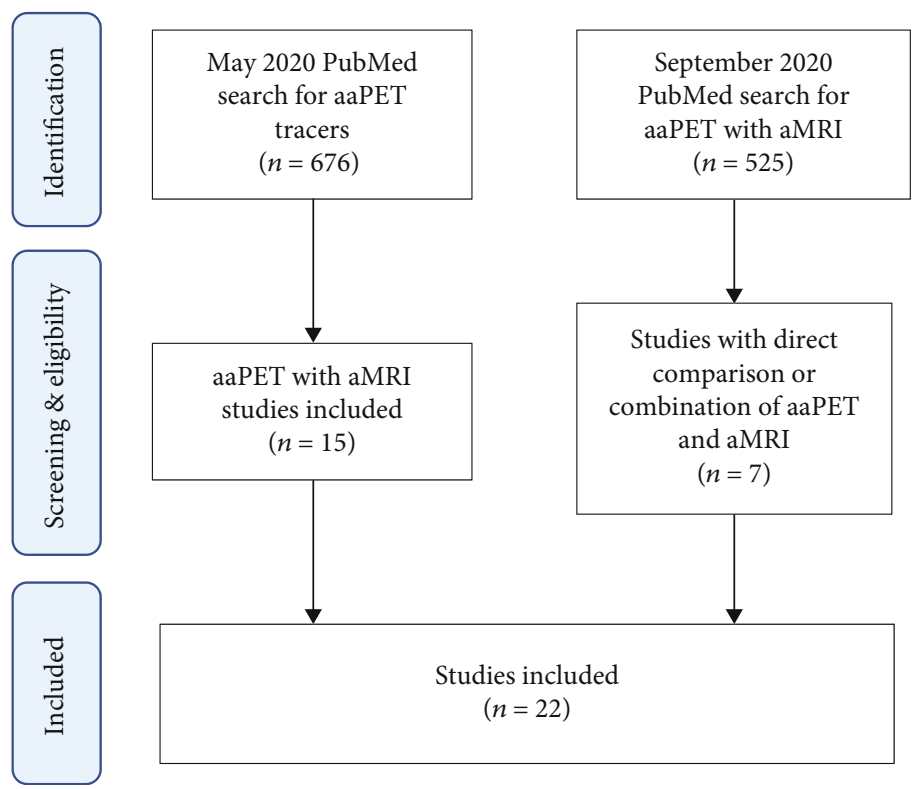

FIGURe 1: PRISMA study selection flowchart.

two that used FDOPA PET [42, 43], three with MET PET [44-46], and two that used FET PET [36, 47], showed mixed results. Overall, there were contradictory results as to whether aaPET uptake was congruent with DWI diffusion coefficient, but aaPET was overall superior for estimating tumor cell density and for differentiating tumor progression from treatment-related changes. Lohmann and colleagues [25] noted that aaPET benefits from robust interinstitution reliability, whereas aMRI is subject to considerable standardization issues. They concluded that the conflicting results of studies reviewed indicate that aaPET and aMRI encode different biological properties, and further research is needed, especially with correlates to neuropathology, to understand how these modalities can be collectively leveraged. Notably, their review did not include the fourth major aaPET tracer, AMT, which has been tested for various neurooncology applications over the past decade.

Overall, there is significant variability in the published results of aaPET and aMRI comparisons, and further investigation of these combined modalities is warranted. In the present review, we summarize the research progress for aaPET and aMRI in for neurooncology imaging, while focusing on reports published in the past 2 years. Specifically, this systematic review considers only those studies that directly compare and/or combine these modalities (aaPET and aMRI). We include the most recent data on the main aaPET tracers and aMRI sequences, as well as some emerging MRI sequences not included in previous review articles.

\section{Methods}

This review included original clinical research studies of either direct comparison of aaPET versus aMRI or combination studies of aaPET with aMRI, in neurooncology applications, published between July 2018 and August 2020. The PubMed database was searched through two mechanisms to identify such publications (Figure 1). This study timeframe was chosen with several months overlap to the previous review, in acknowledgement of the delay between publication and PubMed indexing.

The first search was completed in the end of May 2020, covering the January 2014-May 2020 timeframe, using search terms "amino acid PET brain." A total of 676 records were initially identified using these search terms. These records were then screened for original research publications related to direct comparison of aaPET versus aMRI, or combination study of aaPET with aMRI, in neurooncology, including retrospective and prospective clinical studies, but excluding case reports, review papers, systematic reviews, and meta-analyses. This yielded 15 studies published during the period of July 2018-May 2020.

The second search was completed in September 2020, covering the July 2018-August 2020 timeframe, using the search terms adopted from Lohmann et al. [25]: ((glioblastoma) OR (brain tumors) OR (high-grade glioma)) AND ((positron emission tomography) OR (PET) OR (amino acid PET)) AND ((magnetic resonance imaging) OR (magnetic resonance spectroscopy) OR (perfusion-weighted imaging) OR (diffusion-weighted imaging) OR (chemical exchange saturation transfer) OR (MRI) OR (advanced MRI) OR (MRS) OR (PWI) OR (DWI) OR (CEST) OR (hybrid PET/MR)). This yielded a total of 525 records, which were then screened for original research publications related to direct comparison of aaPET versus aMRI, or combination study of aaPET with aMRI, in neurooncology, including retrospective and prospective clinical studies, but excluding case reports, review papers, systematic reviews, meta-analyses, and papers identified through the first search process. This yielded additional 7 studies, for a total of 22 studies, which were included in the final review (see study selection flowchart in Figure 1 and key study details summarized in Tables 1-4). Data collection and extraction was performed independently by one author (BS) with oversight by the other authors. Data variables collected include year, study location, 
TABLE 1: Summary of aaPET with DWI studies.

\begin{tabular}{|c|c|c|c|c|c|c|c|}
\hline Study & Year & $\begin{array}{c}\text { Study } \\
\text { location }\end{array}$ & $\begin{array}{c}\text { No. of } \\
\text { patients }\end{array}$ & aaPET & DWI & Tumor type & Main results \\
\hline $\begin{array}{l}\text { Castello } \\
\text { et al. }[48]\end{array}$ & 2020 & $\begin{array}{l}\text { Rozzano, } \\
\text { Italy }\end{array}$ & 124 & MET & $\mathrm{ADC}$ & $\begin{array}{l}\text { Operated } \\
\text { gliomas }\end{array}$ & $\begin{array}{l}\text { MET PET is more sensitive for differentiating tumor } \\
\text { grade. Both reach comparable accuracy. }\end{array}$ \\
\hline Popp et al. [47] & 2019 & $\begin{array}{l}\text { Freiburg, } \\
\text { Germany }\end{array}$ & 41 & FET & $\mathrm{ADC}$ & $\begin{array}{l}\text { Recurrent } \\
\text { GBM }\end{array}$ & $\begin{array}{c}\text { FET PET tumor volume is larger than T1w-MRI, which is } \\
\text { larger than DWI. DWI volume only partially overlaps with } \\
\text { FET PET and T1w-MRI, and correlated poorly with } \\
\text { recurrence. }\end{array}$ \\
\hline $\begin{array}{l}\text { Lundemann } \\
\text { et al. [49] }\end{array}$ & 2019 & $\begin{array}{l}\text { Copenhagen, } \\
\text { Denmark }\end{array}$ & 16 & FET & EPI & $\begin{array}{l}\text { Pretreatment } \\
\text { GBM }\end{array}$ & $\begin{array}{l}\text { Combined parameters could map the probability of } \\
\text { recurrence and FET PET more predictive. }\end{array}$ \\
\hline $\begin{array}{l}\text { Werner } \\
\text { et al. [50] }\end{array}$ & 2019 & $\begin{array}{l}\text { Cologne, } \\
\text { Germany }\end{array}$ & 48 & FET & $\mathrm{ADC}$ & $\begin{array}{l}\text { Pretreated } \\
\text { high-grade } \\
\text { glioma }\end{array}$ & $\begin{array}{l}\text { FET PET is superior for differentiating tumor progression. } \\
\text { Combination yielded highest accuracy. }\end{array}$ \\
\hline $\begin{array}{l}\text { Lohmeier } \\
\text { et al. [51] }\end{array}$ & 2019 & $\begin{array}{l}\text { Berlin, } \\
\text { Germany }\end{array}$ & 42 & FET & $\mathrm{ADC}$ & Glioma & $\begin{array}{l}\text { Both reliably differentiate recurrence from posttreatment } \\
\text { changes, but combined provides best diagnostic accuracy. }\end{array}$ \\
\hline $\begin{array}{l}\text { Verburg } \\
\text { et al. [52] }\end{array}$ & 2020 & $\begin{array}{l}\text { Amsterdam, } \\
\text { Netherlands }\end{array}$ & 20 & FET & $\mathrm{ADC}$ & $\begin{array}{l}\text { Newly } \\
\text { diagnosed } \\
\text { glioma }\end{array}$ & $\begin{array}{l}\text { Combination of FET PET with DWI ADC detects } \\
\text { infiltration best in enhancing glioma, and DWI ADC with } \\
\text { T1w-MRI is best in nonenhancing. }\end{array}$ \\
\hline $\begin{array}{l}\text { Piccardo } \\
\text { et al. [54] }\end{array}$ & 2019 & Genoa, Italy & 22 & FDOPA & $\mathrm{ADC}$ & $\begin{array}{l}\text { Pediatric } \\
\text { DMG }\end{array}$ & $\begin{array}{c}\text { Both could differentiate tumor grade, but only FDOPA } \\
\text { PET could differentiate H3K27M-mutant status } \\
\text { independently of histology. }\end{array}$ \\
\hline $\begin{array}{l}\text { Tatekawa } \\
\text { et al. [53] }\end{array}$ & 2020 & $\begin{array}{c}\text { Los Angeles, } \\
\text { USA }\end{array}$ & 63 & FDOPA & $\mathrm{ADC}$ & $\begin{array}{l}\text { Treatment- } \\
\text { naïve glioma }\end{array}$ & $\begin{array}{l}\text { Negatively correlated in IDH-wt and IDH-mutant- } \\
\text { 1p/19q-non-codeleted. Positively correlated in IDH- } \\
\text { mutant-codeleted. }\end{array}$ \\
\hline John et al. [17] & 2019 & Detroit, USA & 30 & AMT & $\mathrm{ADC}$ & $\begin{array}{l}\text { Newly } \\
\text { diagnosed } \\
\text { GBM }\end{array}$ & $\begin{array}{l}\text { Moderate correlation between AMT uptake and ADC } \\
\text { values in nonenhancing tumor regions. Only AMT PET } \\
\text { was associated with overall survival and recurrence. }\end{array}$ \\
\hline
\end{tabular}

number of patients, aaPET tracer(s) studied, aMRI technique(s) studied, tumor type, and main study results. Data was analyzed and summarized qualitatively. Of note, combined PET and MRI scans not acquired simultaneously on a hybrid PET/MRI are deemed to have been performed asynchronously.

\section{Results}

\subsection{Amino Acid PET and Diffusion-Weighted MR Imaging (Table 1)}

3.1.1. MET PET with DWI. MET PET was compared with DWI in a recent study of 124 glioma patients [48]. Scans were performed asynchronously before surgery for 47 high-grade and 77 low-grade gliomas and compared with reference standard histopathology for diagnostic accuracy. DWI had a sensitivity $80.9 \%$, specificity $59.7 \%$, and accuracy (in terms of AUC) $70.3 \%$ for differentiating high- from low-grade glioma, and MET PET had sensitivity 95.7\%, specificity $41.6 \%$, and accuracy of $68.7 \%$. With combined modalities, the authors found that, for DWI-negative gliomas, MET PET demonstrated higher uptake in IDH-1/2wt gliomas. Moreover, both imaging modalities were significant predictors of progression-free survival. Overall, MET PET was found to be more sensitive than DWI for detecting highgrade glioma by visual analysis, though these modalities reached comparable accuracy. Notably, in the patients with negative DWI, the role of MET PET becomes more relevant, perhaps due to the shift in tumor cell density associated with altered water distribution. Although it did not include ADC maps, this multimodality characterization of gliomas builds upon the previous literature, which found MET PET to be more robust than DWI [25], probably reflecting the fact that the modalities encode different biological properties.

3.1.2. FET PET with DWI. FET PET and DWI modalities have been studied jointly in glioma and glioblastoma (GBM) patients in several recent direct comparison reports. In one study of 41 recurrent GBMs, Popp et al. compared tumor volume and localization between contrast-enhanced T1-weighted MRI, FET PET, and DWI, scanned asynchronously [47]. The tumor volumes derived from postcontrast T1-weighted MRI and FET PET were better overlapped with recurrence after reirradiation than those from DWI. The tumor volume from FET PET was larger than that from T1-weighted MRI, which was larger than that of DWI $(P<0.001)$ (Figure 2).

DWI also demonstrated more nonoverlapping area than overlapping area, compared with T1-weighted MRI and FET PET $(P<0.001)$, such that including DWI volume would add up to $48.5 \%$ to the tumor volume [47]. This highlights the complementary nature of these imaging modalities, which capture distinct biological tumor properties, although this study did not correlate the imaging findings with histopathology tissue analysis.

Another small study of 16 GBMs compared FET PET and DWI performed on the same day, for predicting tumor 
TABLE 2: Summary of aaPET with PWI studies.

\begin{tabular}{|c|c|c|c|c|c|c|c|}
\hline Study & Year & $\begin{array}{l}\text { Study } \\
\text { location }\end{array}$ & $\begin{array}{c}\text { No. of } \\
\text { patients }\end{array}$ & aaPET & PWI & Tumor type & Main results \\
\hline $\begin{array}{l}\text { Beppu et al. } \\
{[56]}\end{array}$ & 2019 & $\begin{array}{l}\text { Morioka, } \\
\text { Japan }\end{array}$ & 24 & MET & ASL & Recurrent GBM & $\begin{array}{l}\text { Modalities were significantly correlated before and } \\
\text { after treatment. ASL PWI reliably predicted survival, } \\
\text { but MET PET was more accurate. }\end{array}$ \\
\hline $\begin{array}{l}\text { Pala et al. } \\
\text { [57] }\end{array}$ & 2019 & $\begin{array}{l}\text { Günzburg, } \\
\text { Germany }\end{array}$ & 18 & MET & DSC & GBM & $\begin{array}{l}\text { MET PET is more sensitive than PWI DSC for residual } \\
\text { tumor detection. PWI DSC cannot substitute MET } \\
\text { PET in tumor detection. }\end{array}$ \\
\hline $\begin{array}{l}\text { Qiao et al. } \\
{[58]}\end{array}$ & 2019 & $\begin{array}{l}\text { Beijing, } \\
\text { China }\end{array}$ & 42 & MET & DSC & High-grade glioma & $\begin{array}{l}\text { Both could accurately differentiate radiation injury } \\
\text { from recurrence. Combined, they yielded the best } \\
\text { accuracy. }\end{array}$ \\
\hline $\begin{array}{l}\text { Roodakker } \\
\text { et al. [39] }\end{array}$ & 2019 & $\begin{array}{l}\text { Uppsala, } \\
\text { Sweden }\end{array}$ & 4 & MET & DSC & Oligodendroglioma & $\begin{array}{c}\text { MET PET could identify tumor cell density, but PWI } \\
\text { DSC could not. The modalities were not significantly } \\
\text { correlated. }\end{array}$ \\
\hline $\begin{array}{l}\text { Lundemann } \\
\text { et al. [49] }\end{array}$ & 2019 & $\begin{array}{l}\text { Copenhagen, } \\
\text { Denmark }\end{array}$ & 16 & FET & DCE & Pretreatment GBM & $\begin{array}{l}\text { FET PET had the highest predictive value for } \\
\text { recurrence. Combined parameters could map the } \\
\text { probability of recurrence. }\end{array}$ \\
\hline $\begin{array}{l}\text { Dissaux } \\
\text { et al. [59] }\end{array}$ & 2020 & Brest, France & 30 & FET & DSC & High-grade glioma & $\begin{array}{l}\text { PWI DSC is highly correlated with standard MRI, but } \\
\text { FET PET provides complementary data. }\end{array}$ \\
\hline $\begin{array}{l}\text { Verburg } \\
\text { et al. [52] }\end{array}$ & 2020 & $\begin{array}{l}\text { Amsterdam, } \\
\text { Netherlands }\end{array}$ & 20 & FET & $\begin{array}{l}\text { ASL, } \\
\text { DSC }\end{array}$ & $\begin{array}{l}\text { Newly diagnosed } \\
\text { nonenhancing } \\
\text { glioma }\end{array}$ & $\begin{array}{l}\text { FET PET with PWI DSC together could diagnose } \\
\text { IDH-mutant glioma. }\end{array}$ \\
\hline $\begin{array}{l}\text { Schon et al. } \\
{[60]}\end{array}$ & 2020 & $\begin{array}{l}\text { Munich, } \\
\text { Germany }\end{array}$ & 46 & FET & DSC & $\begin{array}{l}\text { Newly diagnosed } \\
\text { glioma }\end{array}$ & $\begin{array}{l}\text { Both modalities could identify vascularity, but only } \\
\text { FET PET could identify cellularity. }\end{array}$ \\
\hline $\begin{array}{l}\text { Fraioli et al. } \\
{[61]}\end{array}$ & 2020 & London, UK & 40 & FDOPA & DSC & Glioma & $\begin{array}{l}\text { Both could differentiate tumor, but performed better } \\
\text { when combined. FDOPA PET distinguished more } \\
\text { features. }\end{array}$ \\
\hline $\begin{array}{l}\text { Tatekawa } \\
\text { et al. [53] }\end{array}$ & 2020 & $\begin{array}{l}\text { Los Angeles, } \\
\text { USA }\end{array}$ & 61 & FDOPA & DSC & $\begin{array}{l}\text { Treatment-naïve } \\
\text { glioma }\end{array}$ & $\begin{array}{l}\text { Positively correlated in IDH-wt and IDH-mutant- } \\
\text { 1p/19q-non-codeleted. Not correlated in IDH- } \\
\text { mutant-codeleted. }\end{array}$ \\
\hline $\begin{array}{l}\text { John et al. } \\
{[62]}\end{array}$ & 2019 & Detroit, USA & 20 & AMT & DSC & GBM & $\begin{array}{l}\text { Moderate correlation between modalities in } \\
\text { nonenhancing tumor regions. }\end{array}$ \\
\hline
\end{tabular}

TABLE 3: Summary of aaPET with MRS studies.

\begin{tabular}{|c|c|c|c|c|c|c|c|}
\hline Study & Year & $\begin{array}{c}\text { Study } \\
\text { location }\end{array}$ & $\begin{array}{c}\text { No. of } \\
\text { patients }\end{array}$ & aaPET & MRS & Tumor type & Main results \\
\hline $\begin{array}{l}\text { Kudulaiti } \\
\text { et al. [63] }\end{array}$ & 2019 & $\begin{array}{l}\text { Shanghai, } \\
\text { China }\end{array}$ & 109 & MET & $\mathrm{CNI}$ & $\begin{array}{l}\text { Nonenhancing } \\
\text { supratentorial } \\
\text { glioma }\end{array}$ & $\begin{array}{l}\text { MET PET was more sensitive and specific than MRS, } \\
\text { and when combined, sensitivity was higher and } \\
\text { specificity stable. }\end{array}$ \\
\hline $\begin{array}{l}\text { Kebir et al. } \\
{[64]}\end{array}$ & 2019 & $\begin{array}{l}\text { Essen, } \\
\text { Germany }\end{array}$ & 19 & MET & $\begin{array}{l}\text { NAA, } \\
\text { Cho, Cr }\end{array}$ & $\begin{array}{l}\text { Newly } \\
\text { diagnosed } \\
\text { glioma }\end{array}$ & $\begin{array}{l}\text { Both have limited potential in glioma subtyping. MET } \\
\text { PET is better for differentiating IDH status and MRS } \\
\text { for glioma subgrouping. }\end{array}$ \\
\hline $\begin{array}{l}\text { Lundemann } \\
\text { et al. [49] }\end{array}$ & 2019 & $\begin{array}{c}\text { Copenhagen, } \\
\text { Denmark }\end{array}$ & 16 & FET & $\begin{array}{c}\text { 3D } \\
\text { MRSI }\end{array}$ & Glioblastoma & $\begin{array}{l}\text { FET PET had the highest predictive value for } \\
\text { recurrence. 3D MRSI was of insufficient quality for } \\
\text { analysis. }\end{array}$ \\
\hline $\begin{array}{l}\text { Verburg } \\
\text { et al. [52] }\end{array}$ & 2020 & $\begin{array}{l}\text { Amsterdam, } \\
\text { Netherlands }\end{array}$ & 20 & FET & $\mathrm{CNI}$ & $\begin{array}{l}\text { Newly } \\
\text { diagnosed } \\
\text { nonenhancing } \\
\text { glioma }\end{array}$ & $\begin{array}{c}\text { FET PET combined with other modalities detects } \\
\text { glioma infiltration better than standard MRI or FET } \\
\text { PET. MRS data is insufficient due to limited sampling } \\
\text { ability. }\end{array}$ \\
\hline $\begin{array}{l}\text { Piccardo } \\
\text { et al. [54] }\end{array}$ & 2019 & Genoa, Italy & 22 & FDOPA & $\begin{array}{l}\text { CNI, } \\
\text { Cho/Cr, } \\
\text { lactate }\end{array}$ & Pediatric DMG & $\begin{array}{c}\text { Both could differentiate tumor grade and H3K27M } \\
\text { mutation, but only FDOPA PET could do so } \\
\text { independently of histology. }\end{array}$ \\
\hline
\end{tabular}


TABLE 4: Summary of aaPET with emerging aMRI techniques studies.

\begin{tabular}{|c|c|c|c|c|c|c|c|}
\hline Study & Year & $\begin{array}{c}\text { Study } \\
\text { location }\end{array}$ & $\begin{array}{l}\text { No. of } \\
\text { patients }\end{array}$ & aaPET & aMRI & Tumor & Main results \\
\hline $\begin{array}{l}\text { Schon et al. } \\
\text { [60] }\end{array}$ & 2020 & $\begin{array}{l}\text { Munich, } \\
\text { Germany }\end{array}$ & 46 & FET & APT CEST & Newly diagnosed glioma & $\begin{array}{l}\text { APT and FET PET demonstrate overlapping } \\
\text { tumor volume for newly diagnosed glioma. }\end{array}$ \\
\hline Park et al. [68] & 2018 & $\begin{array}{l}\text { Seoul, } \\
\text { Korea }\end{array}$ & 43 & MET & APT CEST & High-grade glioma & $\begin{array}{l}\text { APT outperformed MET PET for } \\
\text { differentiating recurrence. }\end{array}$ \\
\hline $\begin{array}{l}\text { Haubold et al. } \\
\text { [70] }\end{array}$ & 2020 & $\begin{array}{l}\text { Essen, } \\
\text { Germany }\end{array}$ & 42 & FET & $\begin{array}{l}\text { MR } \\
\text { fingerprinting }\end{array}$ & Cerebral glioma & $\begin{array}{l}\text { FET PET with MR fingerprinting could } \\
\text { differentiate tumor grade, ATRX mutation, } \\
\text { IDH1 mutation, and } 1 \mathrm{p} 19 \mathrm{q} \text { codeletion. }\end{array}$ \\
\hline $\begin{array}{l}\text { Shymanskaya } \\
\text { et al. [73] }\end{array}$ & 2020 & $\begin{array}{l}\text { Aachen, } \\
\text { Germany }\end{array}$ & 11 & FET & SISTINA & Cerebral glioma & $\begin{array}{l}\text { SISTINA predicted IDH status better than } \\
\text { FET PET. }\end{array}$ \\
\hline
\end{tabular}

recurrence [49]. FET uptake was the only parameter that could differentiate recurrent tumor volume across all tissue types (white matter, gray matter, contrast-enhancing, and nonenhancing lesions). Other parameters demonstrated tumor discrimination, but their effects were tissuedependent. Combining all PET (FET and ${ }^{18}$ F-fluoro-deoxyglucose [FDG]) and MRI (DWI and PWI) parameters together yielded AUC 0.73 in white matter, 0.78 in gray matter, 0.68 in contrast-enhancing lesions, 0.72 in nonenhancing lesions, and 0.77 overall. They concluded that combining parameters could provide patient-specific maps of probability of recurrence, and FET PET had the highest overall predictive value.

In a study of 48 high-grade gliomas with suspected progression, Werner et al. compared FET PET with DWI for differentiation of treatment-related changes from tumor progression [50]. DWI and FET PET were obtained simultaneously in 11 patients and asynchronously in 37 patients, and diagnosis was confirmed either by neuropathology (79\%) or clinicoradiologically (21\%). FET PET performed better (accuracy 83\%) than DWI (accuracy 69\%) (Figure 3), and when combined, static FET PET plus DWI had an accuracy of $89 \%$, while dynamic FET PET plus DWI had the highest accuracy of $93 \%$. They also observed that FET PET parameters were significant predictors of survival time, whereas DWI was not. Overall, the authors concluded that FET PET should be preferred over DWI for differentiating tumor progression form treatment-induced tissue changes [50].

Similarly, Lohmeier et al. studied the comparative ability of FET PET and DWI to differentiate between recurrent glioma and treatment-related effects in 42 glioma patients [51]. Using simultaneous PET/MRI acquisition, they found that both modalities have reliable diagnostic performance: FET PET (AUC 0.81, sensitivity $81 \%$, and specificity $60 \%$ ) and DWI (AUC 0.82, sensitivity 62\%, and specificity $100 \%)$. Their combined performance in a biparametric approach had the highest diagnostic accuracy (AUC 0.90, sensitivity $97 \%$, and specificity $60 \%$ ), though there was no statistically significant difference in diagnostic power. The benefit of combining modalities was especially important when the FET PET parameter of tumor-to-brain ratio maximum was close to threshold, in which case the addition of the DWI parameter ADC-mean effectively improved clinical detection.
In Verburg et al., 20 newly diagnosed gliomas were scanned with FET PET and DWI asynchronously before treatment [52]. They compared the ability of each imaging modality to detect tumor as compared to neuropathology confirmation. For nonenhancing gliomas, DWI with T1weighted MRI yielded the best tumor detection (AUC 0.90) and had the highest prediction accuracy (88\%). FET PET was not included in the optimal combination for nonenhancing glioma and in fact had lower diagnostic accuracy than standard fluid-attenuated inversion recovery (FLAIR) MRI. Further subtype analysis found that the highest accuracy for diagnosing high-grade IDH-wild type (IDH-wt) FETpositive glioma was using DWI combined with FET PET (AUC 0.89), and for FET-negative gliomas, the DWI parameters had the highest diagnostic accuracy (AUC 1.00). They conclude that enhancing glioma infiltration is best detected by a combination of DWI with FET PET, and importantly, although FET PET is part of the optimal imaging combination for most brain tumors, it is not for nonenhancing gliomas. This finding is unique among FET PET with DWI studies, which largely support FET PET as the superior imaging modality, because with nonenhancing gliomas, DWI performs better.

3.1.3. FDOPA PET with DWI. In a study of treatment-naïve gliomas, Tatekawa et al. imaged 63 patients with DWI and FDOPA PET, within 2 months of each other [53]. They found a negative correlation between FDOPA PET SUV and DWI ADC in IDH-wt gliomas, both on voxel-wise $(r=-0.19)$ and patient-wise $(r=-0.58)$ analysis. There was a negative correlation in IDH-mutant-1p/19q-non-codeleted $\left(\mathrm{IDH}_{\mathrm{m} \text {-non-codel }}\right)$ gliomas, both on voxel-wise $(r=-0.19)$ and patient-wise $(r=-0.61)$ analysis. In IDH-mutant-codeleted $\left(\mathrm{IDH}_{\mathrm{m} \text {-codel }}\right)$ gliomas, there was a positive correlation only on voxel-wise analysis $(r=0.18)$. The $r$ value on voxel-wise analysis was significantly higher in $\mathrm{IDH}_{\mathrm{m} \text {-codel }}$ than in IDHwt or $\mathrm{IDH}_{\mathrm{m} \text {-non-codel }}(P<0.001)$, but it was not significantly different between molecular groups on patient-wise analysis. On receiver operating curve (ROC) analysis, $\mathrm{IDH}_{\text {m-codel }}$ was differentiated from IDH-wt and $\mathrm{IDH}_{\mathrm{m} \text {-non-codel }}$ with an AUC of 0.80 (sensitivity 63\% and specificity 92\%). On Cox multivariate analysis of IDH-wt glioma, there was a significant voxel-wise association ( $\mathrm{HR}=0.085, P=0.038)$. And on the log-rank tests of IDH-wt gliomas, there was a 


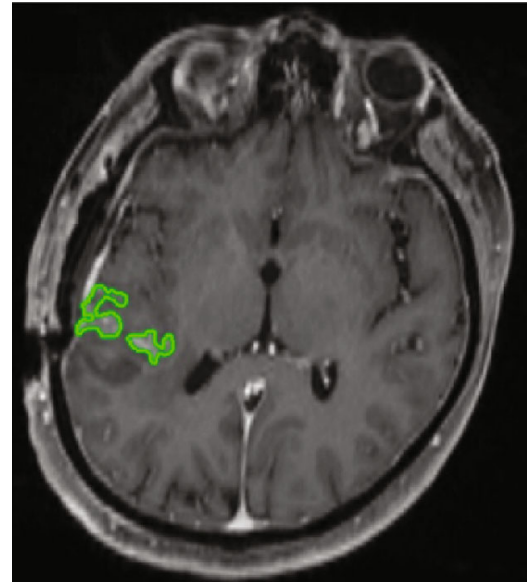

(a)

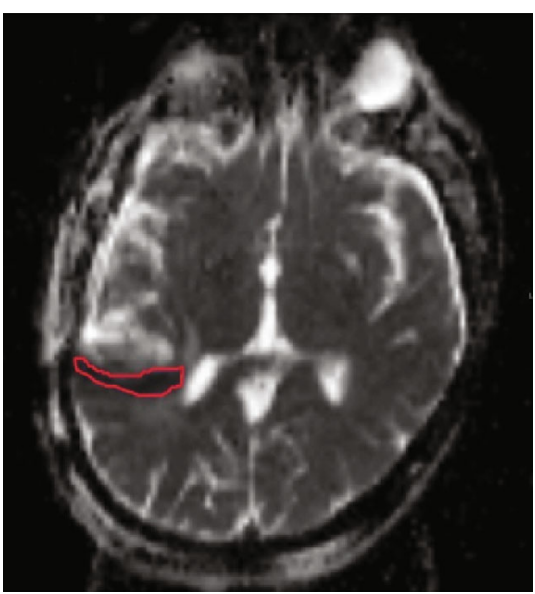

(c)

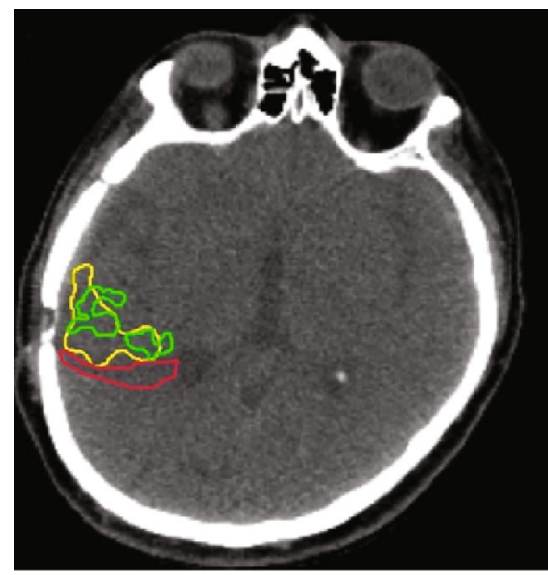

(e)

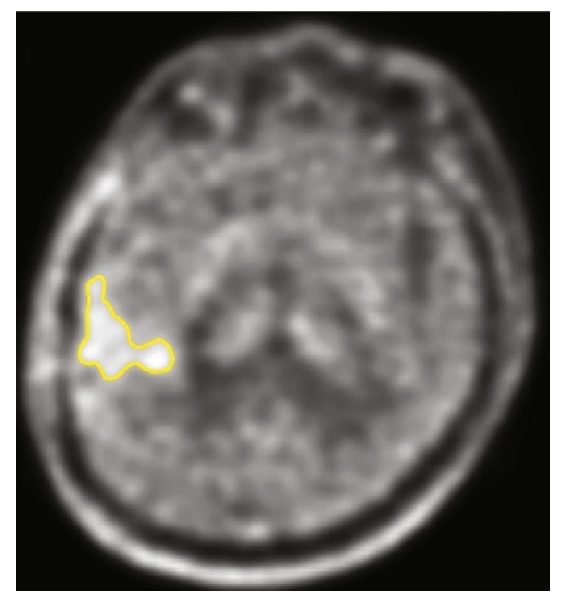

(b)

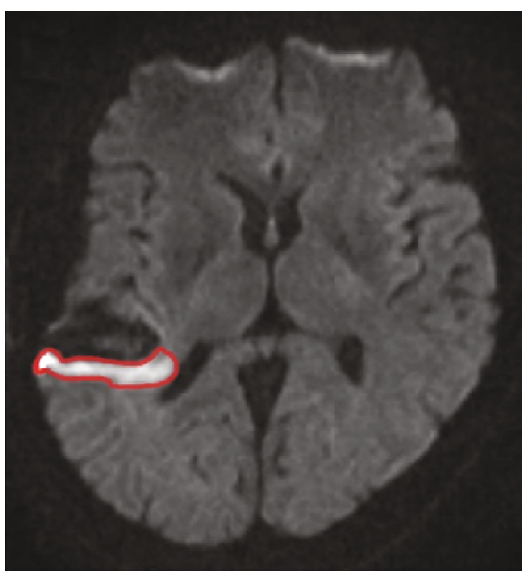

(d)

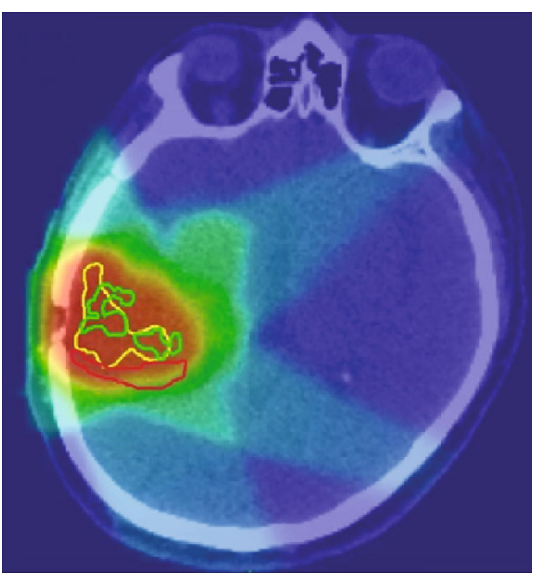

(f)

FIGURE 2: FET PET combined with DWI in recurrent glioblastoma (histologically confirmed). (a) Axial postcontrast T1-weighted MRI, green: gross total volume (GTV). (b) Axial FET PET, yellow: GTV on PET. (c) ADC map, red: GTV by ADC (low), corresponding to a hyperintensity in (d) DWI-MRI. (e) Illustration of all three volumes on planning CT. (f) Dose distribution of the treatment plan based on FET PET. The volumetric comparison thus revealed that the majority of the GTVs-ADC (low) is located outside areas of contrast enhancement in postcontrast T1-weighted MRI (mean nonoverlapping volume $66.2 \pm 24.6 \%$, median $72.2 \%$, and range 5.8-100\%) and also outside areas of increased FET uptake (mean nonoverlapping volume $76.4 \pm 64 \%$, median $69.5 \%$, and range $12.3-385.1 \%$ in the total cohort, $n=41$ ). Reproduced with permission from Figure 3 in Popp et al. [47].

significant difference in overall survival when IDH-wt glioma was stratified by the voxel-wise $r$ value, with lower $r$ value being associated with worse survival [53].
Piccardo et al. scanned 22 pediatric diffuse midline gliomas (DMGs) with an asynchronous multimodal imaging approach [54]. They demonstrated a strong correlation 


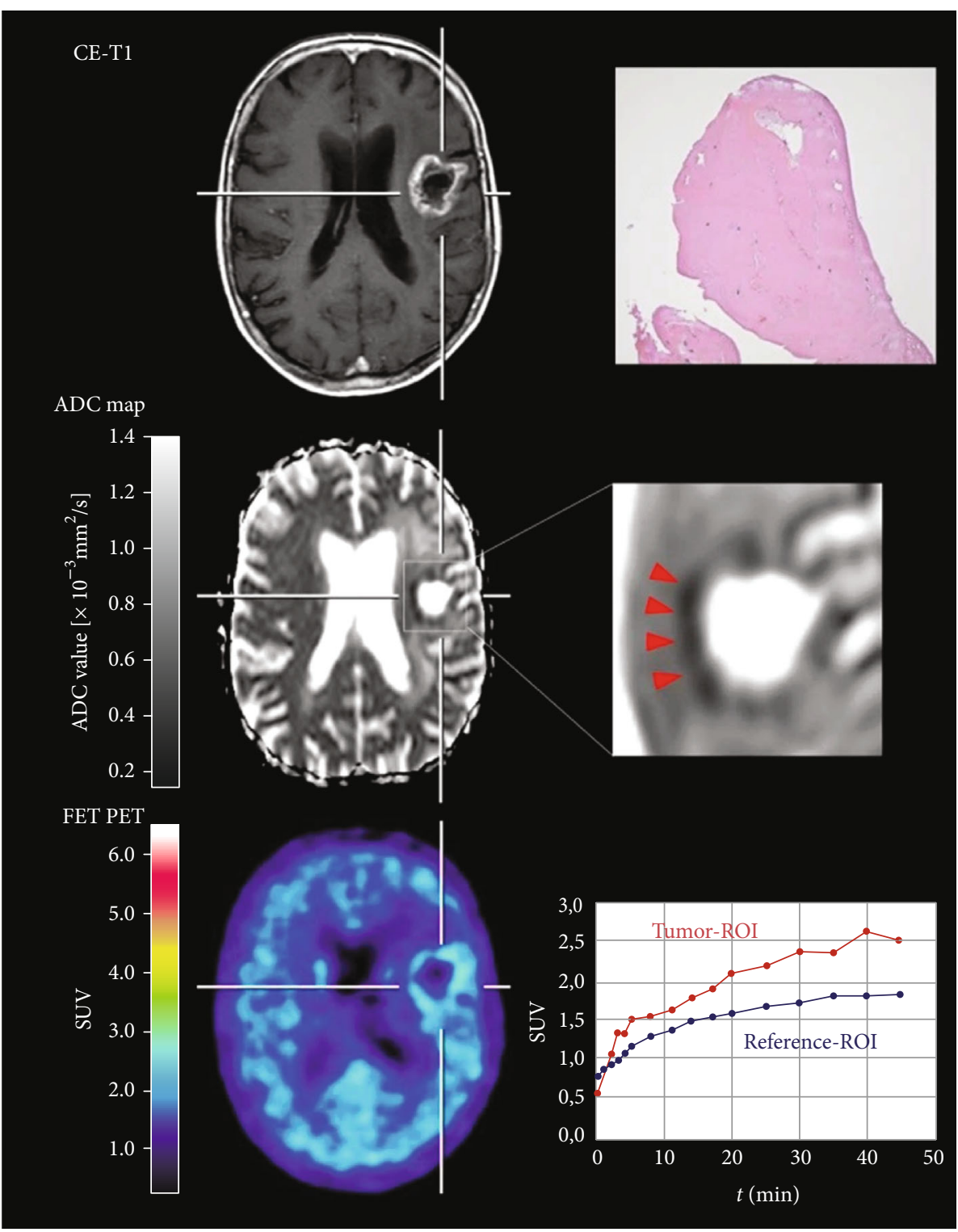

FIGURE 3: FET PET combined with DWI to detect radiation necrosis (histologically confirmed) after glioma treatment. Contrast-enhanced MRI, ADC map obtained from DWI, and FET PET of a 69-year-old female patient with anaplastic oligodendroglioma. Twenty-nine months after fractionated external beam radiotherapy, brachytherapy, and adjuvant temozolomide chemotherapy, contrast-enhanced MRI suggested tumor progression. In spatial correspondence to the contrast enhancement, the ADC map revealed a substantial decrease of diffusivity in the area of contrast enhancement (arrowheads on the enlarged image; ADC below $0.5 \times 10^{-3} \mathrm{~mm}^{2} / \mathrm{s}$ ), suggesting tumor progression. In contrast, FET PET showed no increased metabolic activity and a steadily increasing time-activity curve, indicating treatment-related changes. Histological findings obtained following stereotactic biopsy were consistent with radiation necrosis (hematoxylin and eosin staining: original magnification, $\times 200$; scale bar, $50 \mu \mathrm{m}$ ). For a follow-up time of 6 years, the patient was in a stable clinical condition. Reproduced with permission from Figure 1 of Werner et al. [50].

between FDOPA tumor-to-striatum uptake ratio and DWI relative minimum $\mathrm{ADC}(P<0.01)$. Both parameters were able to successfully differentiate low- and high-grade DMGs $(P<0.01)$ : FDOPA PET tumor-to-striatum ratio provided AUC 0.94 (sensitivity $83 \%$ and specificity 60\%), FDOPA PET tumor-to-brain ratio gave AUC 0.82 (sensitivity $91 \%$ and specificity $80 \%$ ), while DWI relative minimum ADC yielded AUC 0.81 (sensitivity $83 \%$ and specificity $70 \%$ ). However, the only parameter to significantly differentiate between H3K27M-mutant and wild-type DMGs indepen- dent of histology was FDOPA uptake ratio $(P=0.003)$. DWI did not reach significance for differentiating H3K27M-mutant status $(P=0.21)$ [54].

3.1.4. AMT PET with DWI. A comparative study of AMT PET with DWI, by John et al., scanned 30 newly diagnosed GBMs, with an average 3 days between DWI MRI and AMT PET scans [17]. They identified a strong negative correlation between AMT standardized uptake values (SUVs) and DWI-derived ADC $(P<0.0001)$ (Figure 4). Areas 


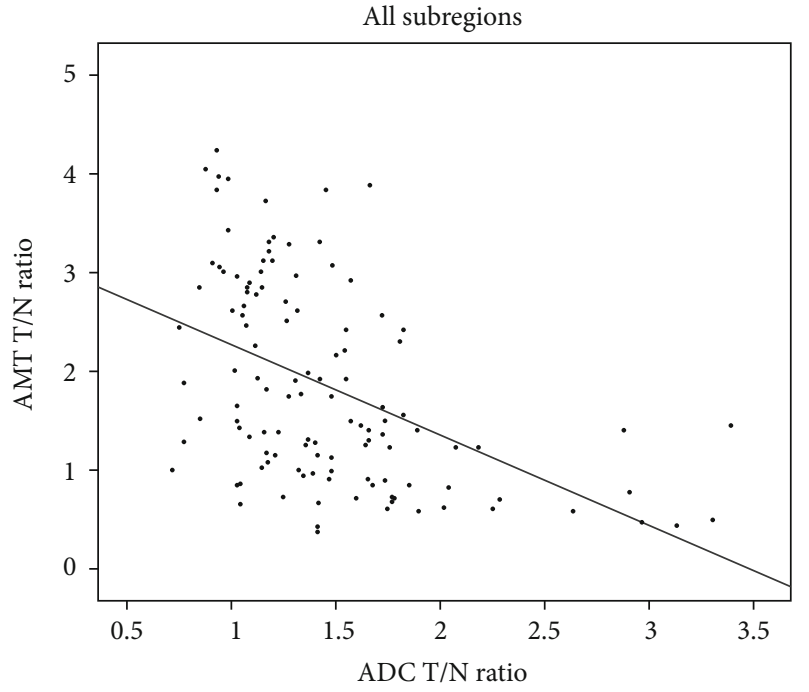

(a)

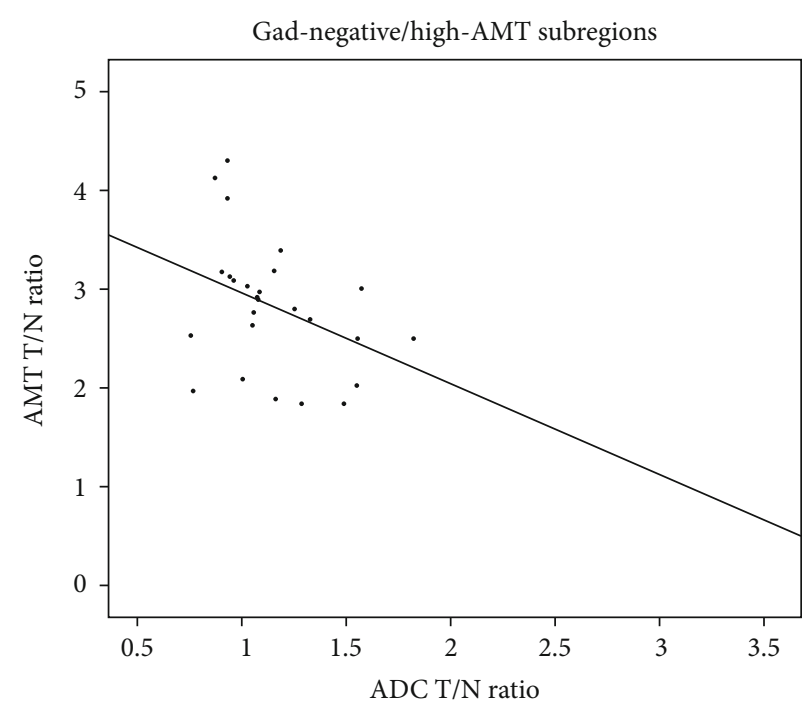

(b)

FIgURE 4: Correlation between AMT uptake and ADC values in glioblastoma subregions. (a) Negative correlation in the whole set of tumor subregions $(N=115$, including tumor regions with both high and low AMT uptakes; Spearman's rho $[r]=-0.52, P<0.0001)$. (b) A similar trend was detected in noncontrast-enhancing/high-AMT tumor subregions, consistent with tumor-infiltrated brain $(N=25, r=-0.40, P$ $=0.05$ ). Reproduced with permission from Figure 3 of John et al. [17].

beyond contrast enhancement on MRI that demonstrated high AMT PET uptake were associated with low ADC on DWI $(P=0.05)$, and these areas were indicative of tumorinfiltrated brain. In nonenhancing T2w/FLAIR hyperintense areas, there was also a correlation between low AMT PET uptake with high ADC values, which indicates peritumoral vasogenic edema. Only AMT PET uptake was associated with overall survival (HR 7.8, $P=0.003$ ), and high uptake ratio was also predictive of the location of posttreatment tumor progression [17].

This study built on a previous study of the same group that combined AMT PET with DWI-derived isotropic diffusion spectrum imaging, which detected metabolically active glioma regions with high cellularity and differentiated highvs. low-grade gliomas accurately albeit in a small cohort $(n=10)[55]$.

\subsection{Amino Acid PET and Perfusion-Weighted MR Imaging (Table 2)}

3.2.1. MET PET with PWI. MET PET imaging was compared to PWI MRI in several studies over the past two years. Beppu et al. scanned 24 recurrent GBMs asynchronously using MET PET and ASL perfusion imaging before and 4 and 8 weeks after treatment initiation [56]. MET PET tumor volume was significantly larger than that of ASL at baseline, but no significant difference was found after treatment. MET PET and ASL parameters were significantly correlated at all time points. Each modality demonstrated moderate predictive power for progression-free survival: MET PET had AUC 0.44 at baseline, 0.66 at 4 weeks, and 0.73 at 8 weeks; ASL had AUC 0.41 at baseline, 0.65 at 4 weeks, and 0.66 at 8 weeks. ASL could predict progression-free survival, but the most accurate predictor was MET PET, with $76.9 \%$ sensitivity and $81.8 \%$ specificity at 8 weeks. The authors concluded that these modalities provide different information; however, they did not study the performance of these imaging modalities combined [56]. In another study of 18 GBMs, Pala et al. obtained MET PET and PWI scans asynchronously before, during (MRI only), and after surgery in order to compare tumor volume and diagnostic accuracy compared to histopathology [57]. Similar to Beppu et al., they found that before gross total resection, MET PET yielded a larger tumor volume than PWI, a difference that disappeared after surgery. MET PET demonstrated higher sensitivity for GBM detection (95\%) than PWI (67\%). The authors did not explore the question of combining modalities [57].

Comparing MET PET to DSC PWI for their ability to differentiate radiation injury from recurrent tumor, Qiao et al. scanned 42 high-grade gliomas asynchronously [58]. All parameters were shown to differ significantly between recurrent tumor tissue and radiation injury tissue. MET PET yielded AUC 0.847 , sensitivity $90.9 \%$, and specificity $55.6 \%$, while PWI yielded AUC 0.845, sensitivity 66.7\%, and specificity $77.8 \%$. Combining imaging modality parameters resulted in the largest AUC, 0.953, with sensitivity $84.8 \%$ and specificity $100 \%$ (Figures 5 and 6). They concluded that the combination of these modalities yielded the best diagnostic accuracy to differentiate recurrence from radiation injury in high-grade glioma [58].

Studying these imaging modalities in oligodendrogliomas, Roodakker et al. compared MET PET and PWI images acquired asynchronously, with histology as the ground truth, in four oligodendrogliomas [39]. MET PET uptake was significantly correlated to tumor cell density, but PWI was not correlated with MET PET or histology. They concluded that MET PET, but not PWI, has a value as an indicator of tumor cell density in oligodendrogliomas. While in a very small study, this result differs from that of the GBM studies above and may indicate an important tumor type difference, 


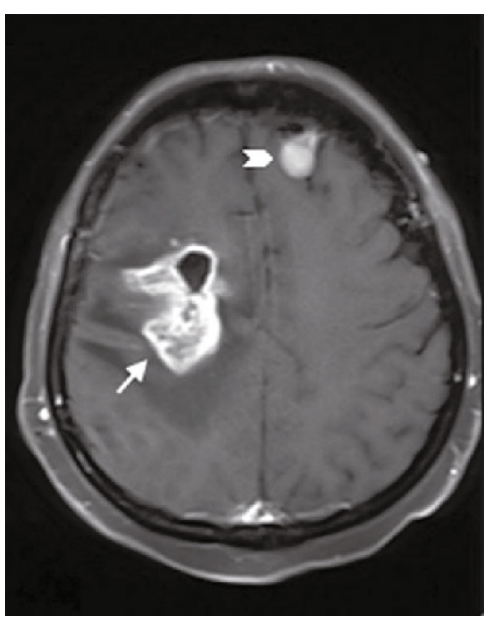

(a)

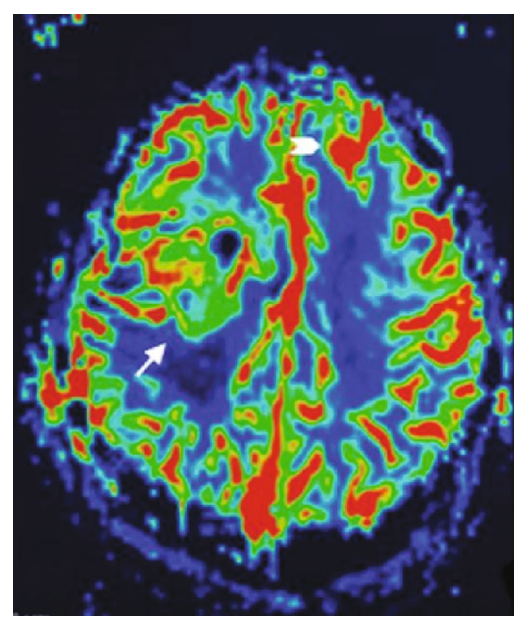

(b)

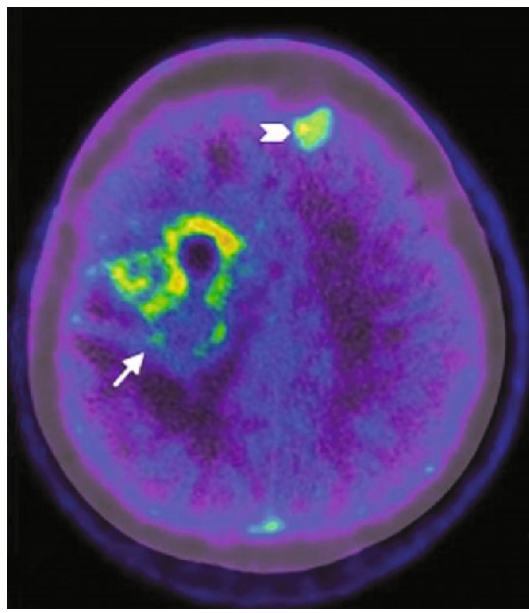

(c)

FIgURE 5: MET PET combined with PWI in recurrent glioblastoma. Contrast-enhanced T1-weighted MR image (a) and a relative cerebral blood volume (CBV) map (b) and a MET PET/CT image (c) for a 41-year-old woman with recurrent glioblastoma. The lesion in the right frontal lobe (arrow) shows enhancement (a) and positive findings on both the rCBV map (b) and MET PET/CT image (c). The lesion localized in the left frontal lobe (chevron) might be a meningioma, which has been stable for several years. Reproduced with permission from Figure 2 of Qiao et al. [58].

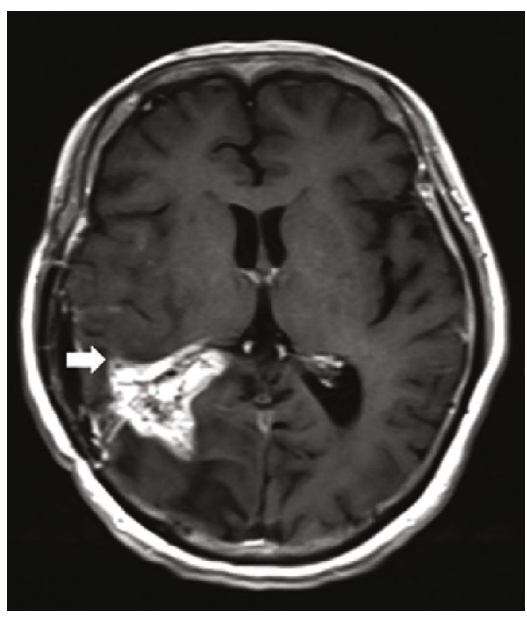

(a)

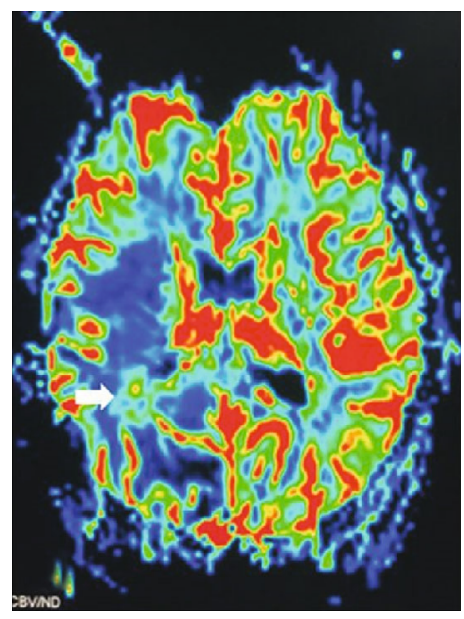

(b)

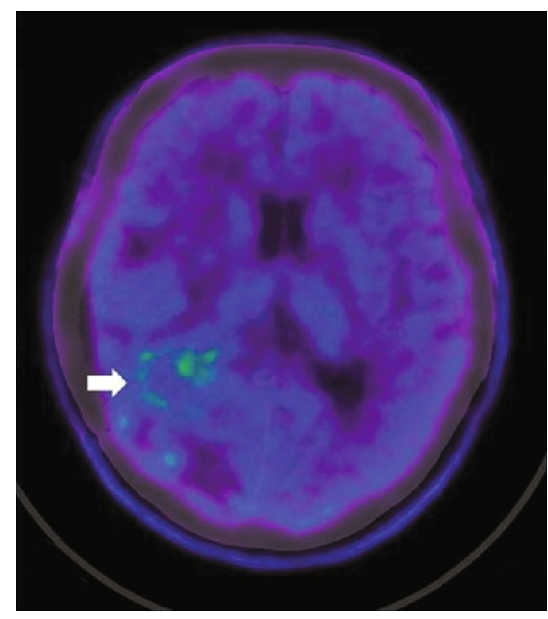

(c)

FIgURE 6: MET PET with PWI of radiation necrosis. Contrast-enhanced T1-weighted MR image (a) and relative CBV map (b) and MET $\mathrm{PET} / \mathrm{CT}$ image (c) for a 44-year-old man with necrosis. The lesion in the right parietal lobe (arrow) shows enhancement (a) and negative findings on both the rCBV map (b) and the MET PET/CT image (c). Reproduced with permission from Figure 3 of Qiao et al. [58].

wherein the value of PWI may be negligible for oligodendrogliomas specifically [39].

3.2.2. FET PET with PWI. Advances have also been made in comparing FET PET to PWI in the past two years. Lundemann et al. compared FET PET and PWI for predicting recurrence in 16 GBMs [49]. FET uptake was the only parameter that could significantly differentiate recurrence in all tissue types, while PWI parameters had a tissuedependent effect, in white matter, gray matter, and nonenhancing lesion. When combined, FET PET, DWI, PWI, and FDG PET/MRI parameters together achieved AUC 0.73 in white matter, 0.78 in gray matter, 0.68 in contrast- enhancing lesions, 0.72 in nonenhancing lesions, and 0.77 overall. They concluded that FET PET had the strongest predictive value for recurrence in GBM [49].

FET PET and PWI have been investigated in high-grade gliomas as well. Dissaux et al. assessed these modalities asynchronously in 30 patients, to assess tumor volume delineation compared to standard MRI [59]. Using scans obtained an average of 6 days apart, they found that FET PET tumor volume was significantly larger than volume on standard MRI $(P=0.005)$ with a low overlap volume, whereas PWI volume was significantly smaller than standard MRI volume $(P<0.001)$ with a high overlap volume. They concluded that PWI parameters were highly correlated with standard MRI, 
whereas FET PET provided complementary information. This analysis is limited by the lack of histologic confirmation and the lack of direct comparison or combination analysis of FET PET with PWI [59].

Verburg et al., in their multimodal imaging analysis of 20 newly diagnosed gliomas, compared FET PET with PWI acquired asynchronously before treatment, to compare diagnostic accuracy [52]. In IDH-mutant gliomas, FET PET combined with DSC PWI yielded an AUC 0.82. They did not report on this imaging combination for any other tumor subtypes. In another study of 46 newly diagnosed gliomas, Schon et al. [60] found the tumor volume to be larger by FET PET than by DSC PWI. For identifying cellularity, FET PET parameters performed the strongest, while for vascularity, both DSC PWI and FET PET performed well. The authors noted that in FLAIR hyperintense GBM regions, PWI parameters were significantly higher in the FET PETpositive areas $(P<0.001)$ [60].

Similar to the previous literature, which found variability in spatial correlation of aaPET with PWI, these new data also show inconsistent results. In high-grade gliomas, there was little overlapping volume between these imaging modalities, but in newly diagnosed gliomas, the high FET PET uptake regions had increased PWI parameters. There may be tumor type differences or PWI sequence differences contributing to these discrepancies.

3.2.3. FDOPA PET with PWI. In a study of 40 gliomas, Fraioli et al. scanned patients with FDOPA PET and DSC PWI synchronously, with tumor confirmed on biopsy, to assess the ability of these modalities to identify tumor features [61]. PWI parameters correlated with tumor grade $(P<0.001)$ but not with FDOPA PET parameters. FDOPA PET maximum SUV analysis was able to distinguish enhancing and nonenhancing tumor components from necrosis and normal tissue. FDOPA PET and PWI each achieved an AUC 0.94 for differentiating tumor tissue, and when combined, they achieved a high AUC of 0.99 .

In a study of treatment-naïve gliomas, Tatekawa et al. imaged 61 patients with PWI and FDOPA PET, within 2 months of each other [53]. They found in IDH-wt gliomas a positive correlation between FDOPA PET SUV and PWI relative cerebral blood flow (rCBV), both on voxel-wise $(r=0.25)$ and patient-wise $(r=0.50)$ analysis. There was a positive correlation in $\mathrm{IDH}_{\mathrm{m} \text {-non-codel }}$ gliomas, both on voxel-wise $(r=0.31)$ and patient-wise $(r=0.70)$ analysis. There was no correlation in $\mathrm{IDH}_{\mathrm{m} \text {-codel }}$ gliomas. The $r$ value on voxel-wise analysis was significantly lower in $\mathrm{IDH}_{\mathrm{m} \text {-codel }}$ than in IDH-wt or $\mathrm{IDH}_{\text {m-non-codel }}(P<0.01)$, but it was not significantly different between molecular groups on patientwise analysis. On ROC analysis, $\mathrm{IDH}_{\mathrm{m} \text {-codel }}$ was differentiated from IDH-wt and $\mathrm{IDH}_{\mathrm{m} \text {-non-codel }}$ with an AUC of 0.68 (sensitivity $69 \%$ and specificity $73 \%$ ). Weak voxel-wise correlation between FDOPA SUV and $\mathrm{rCBV}$ or ADC had a significant association with better overall survival in IDHwt gliomas. The authors explained this by a mechanism where gliomas with heterogeneous features show weak correlations between physiological MRI/PET, have less efficient growth, and have better prognosis.
3.2.4. AMT PET with PWI. In the first comparative study of AMT PET with PWI, John et al. scanned 20 GBMs asynchronously with an average of 3 days between scans to evaluate characteristics of the nonenhancing tumor regions [62]. AMT PET and PWI demonstrated a moderate positive correlation in T2w/FLAIR hyperintense regions $(r=0.41$ and $P$ $=0.017)$, which was stronger for recurrent tumors $(r=0.55$ and $P=0.034)$ than for newly diagnosed GBM $(r=0.23$ and $P=0.37)$. This demonstrates an overall moderate correlation between AMT PET and PWI in nonenhancing GBM regions, and a spatial mismatch between AMT uptake and relative cerebral blood volume was often present (Figure 7).

Tumor regions with very low rCBV (below 0.79 tumor/normal ratios), measured by PWI, showed invariably low AMT uptake. AMT PET was able to detect metabolically active tumor portions in nonenhancing (T2w/FLAIR hyperintense) tumor regions if the $\mathrm{rCBV}$ values were above the 0.79 threshold [62].

\subsection{Amino Acid PET and MR Spectroscopy (Table 3)}

3.3.1. MET PET with MRS. Two recent studies compared MET PET with MRS. Kudulaiti et al. retrospectively reviewed the scans of 109 nonenhancing supratentorial lesions with MET PET and MRS prior to treatment, and compared their ability to differentiate glioma from nonglioma lesions [63]. The Cho/NAA index (CNI) for each voxel was used as the MRS parameter. Pathology revealed that the most common tumor types were astrocytoma (38.5\%), oligodendroglioma $(16.5 \%)$, and anaplastic astrocytoma (16.5\%). MRS reached a sensitivity of $60 \%$ and specificity of $50 \%$, whereas MET PET reached a higher sensitivity of $75.8 \%$ and a specificity of $50 \%$, a statistically significant difference. With both PET and MRS combined, the sensitivity rose to $89.5 \%$, which was significantly higher, and specificity dropped to $42.9 \%$, which was not significant.

Kebir et al. scanned 19 histologically confirmed gliomas with MET PET and MRS in a single standardized imaging session, prior to treatment, and compared their ability to classify glioma molecular subtypes [64]. The spectroscopy peaks for NAA, choline, and $\mathrm{Cr}$ were used as the MRS parameters. MET PET uptake was superior for identifying IDH status (AUC 0.67), with a tumor-to-brain uptake ratio higher in IDH-wt gliomas (3.61) than in IDH-mutant gliomas (2.37). MRS was superior for glioma subgrouping of IDH-wt GBM, IDH-wt grade II/III glioma, and IDHmutant grade II/III glioma with and without $1 \mathrm{p} / 19 \mathrm{q}$ codeletion (AUC 0.68). These modalities performed better individually, as the combined modalities yielded AUC 0.61 for classifying glioma subtypes and 0.58 for classifying IDH status. Together, these studies show that MET PET and MRS individually and together have diagnostic potential for diagnosing glioma, and differentiating high-grade from lowgrade, but that they perform relatively poorly for classifying glioma molecular subtype. This extends upon the previous literature, which reported that both MET PET and MRS hold high diagnostic accuracy for diagnosing high-grade glioma recurrence. 


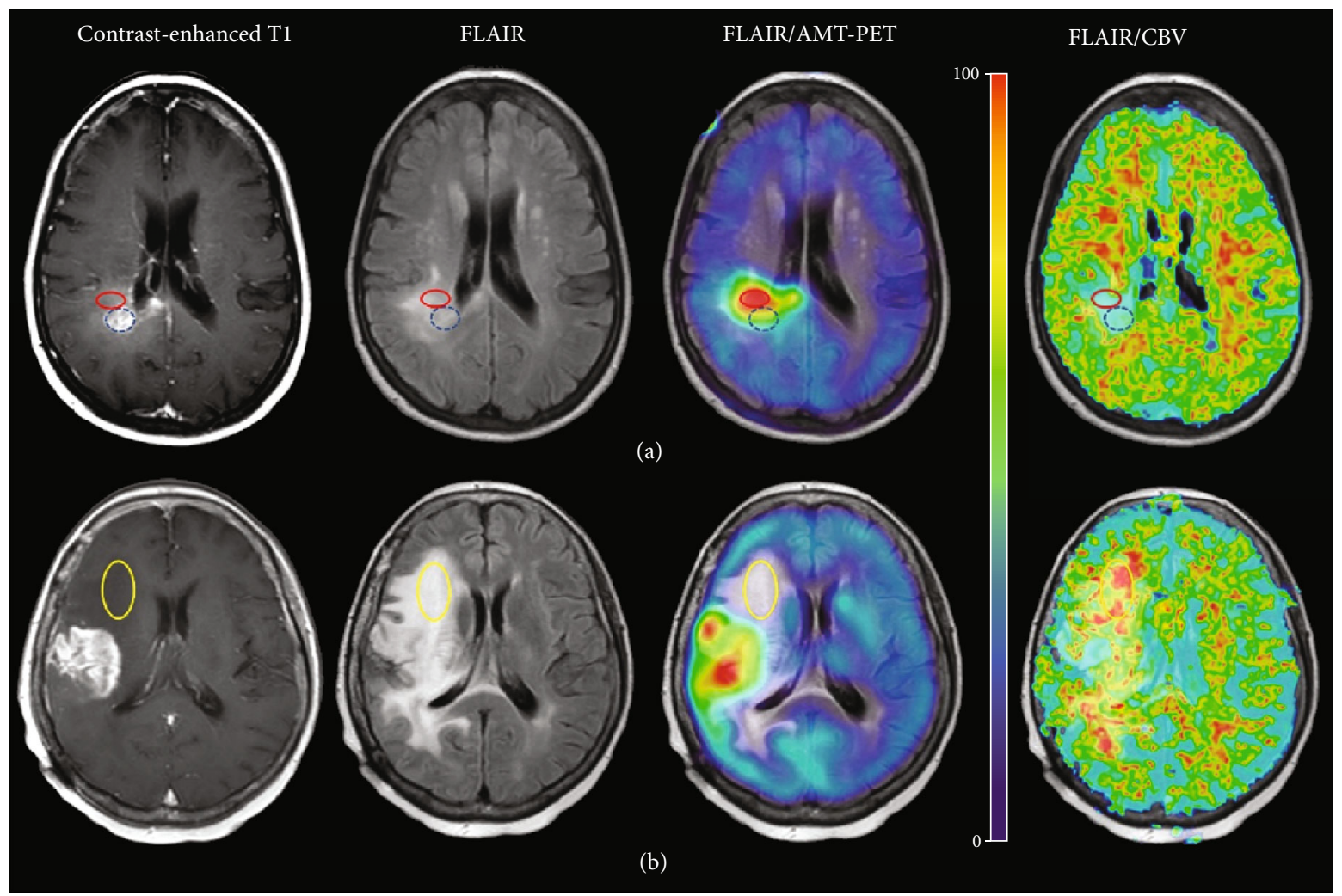

FIGURE 7: Spatial mismatch between AMT PET uptake and relative cerebral blood volume (CBV) on PWI. Examples of ${ }^{11} \mathrm{C}$-alpha-methyl-Ltryptophan (AMT) and relative cerebral blood volume (rCBV) mismatch in patients with newly diagnosed (a) and recurrent (b) glioblastoma. (a) In patient \#6, high AMT uptake (AMT tumor to normal [T/N] ratio: 2.78) was associated with low rCBV values (T/N ratio: 0.88 ) (red circles) adjacent to the contrast-enhancing tumor mass (blue dashed circles). (b) In patient \#15, low AMT uptake (T/N ratio: 0.54 ) was associated with increased $\mathrm{rCBV}$ values (T/N ratio: 1.60) in the extensive fluid-attenuated inversion recovery (FLAIR) hyperintense area surrounding the contrast-enhancing tumor mass with high-AMT uptake (yellow circle). The color bar shows a relative scale (0\%-100\%). Reproduced with permission from Figure 3 of John et al. [62].

3.3.2. FET PET with MRS. Recent comparisons of MRS data with FET PET have been less fruitful. There were two studies that combined FET PET with MRS; however, one study attempted 3D MRS imaging with a quality insufficient for analysis [49], while the other study found that imaging measurements of MRS were missing from a large number of biopsy samples as the voxels did not include brain regions where the biopsy was originated from [52]. As such, there are no new successful advances in FET PET with MRS, contrary to the review by Lohmann et al. [25], which found several reports supporting the conclusion that FET uptake was significantly correlated with MRS metrics, although they were spatially incongruent and represented complementary information. It may be that because both recent studies were taking a multimodal imaging analysis approach, the relatively weak or incomplete data from MRS were excluded in favor of focusing on the other stronger modalities. Future research efforts focused specifically on the utility of wholebrain MRS imaging [65], providing more widespread sampling ability, with aaPET may be able to overcome such limitations.

3.3.3. FDOPA PET with MRS. FDOPA PET has also been studied alongside MRS in a recent retrospective review of 22 pediatric DMGs [54]. In this multimodal imaging study, the authors compared, among others, FDOPA PET to MRS using scans obtained two weeks apart and compared the diagnostic potential of each modality to histology and molecular analysis. The MRS parameters used were choline-tocreatine peak area ratio, choline-to-N-acetylaspartate peak area ratio, and lactate peak. FDOPA PET and MRS parameters were both able to differentiate low-grade from highgrade DMG and to differentiate between H3K27M-mutant and wild-type DMG (Figure 8).

FDOPA PET outperformed MRS on ROC analysis, with AUC 0.94 compared to 0.78 , respectively. FDOPA uptake was the only parameter that could discriminate H3K27Mmutant from wild-type DMG independently of histology (AUC 0.91). Overall, FDOPA PET was more diagnostic for pediatric DMG, although they did not attempt to combine modalities for improved diagnostic ability. Future research efforts may thus be targeted towards comparing these modalities in different tumor types and exploring the possibility of improved diagnostics with combined imaging parameters.

3.4. Emerging aMRI Techniques Combined with aaPET (Table 4). There are several aMRI imaging techniques that are emerging in response to the need for improved neuroimaging. These techniques are not well-developed or researched enough to include in the above discussions, but we include 


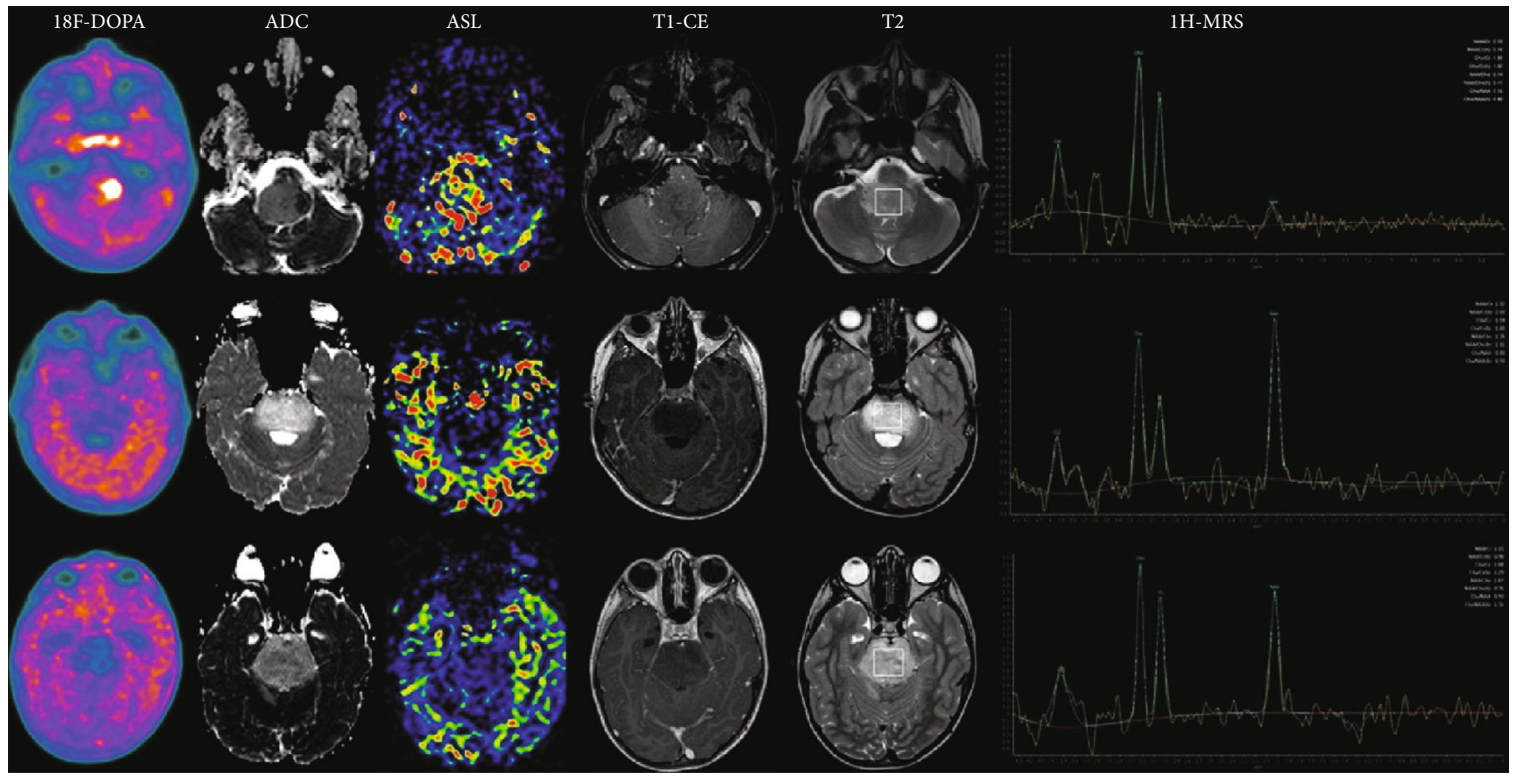

FIGURE 8: FDOPA PET combined with MRS in diffuse midline glioma (DMG). ${ }^{18}$ F-DOPA PET and MR images of H3K27M-mutant and wild-type DMG. Upper row: DMG, H3K27M-mutant, and WHO grade IV; ${ }^{18} \mathrm{~F}$-DOPA PET shows an area of markedly increased uptake within the lesion (tumor/normal [T/N] ratio: 2.80; tumor/striatum [T/S] ratio: 1.80). ADC demonstrates a focal area with mildly reduced diffusivity (rADC min: 0.95 ) on the left side of the lesion corresponding to the ${ }^{18} \mathrm{~F}$-DOPA PET hot spot region. ASL shows increased perfusion (rCBF max: 1.90). Contrast-enhanced (CE) T1-weighted MR image does not show contrast enhancement. ${ }^{1} \mathrm{H}-\mathrm{MRS}$ demonstrates marked increase of Cho/NAA (7.15) and mild increase of Cho/Cr (1.39) ratios. Middle row: diffuse intrinsic pontine glioma, H3K27M-wild type, and WHO grade III; ${ }^{18} \mathrm{~F}-\mathrm{DOPA}$ PET shows absence of tracer uptake in the lesion (T/N: 1.00; T/S: 0.60). ADC demonstrates increased diffusion (rADC min: 1.42). ASL shows low perfusion (rCBF max: 0.80). CE T1-weighted MR image does not show contrast enhancement. ${ }^{1} \mathrm{H}-\mathrm{MRS}$ demonstrates normal Cho/NAA (0.80) and mild increase of Cho/Cr (1.54) ratios. Of note, this was the only histologically defined high-grade glioma which demonstrated lack of increased ${ }^{18} \mathrm{~F}$-DOPA uptake. Lower row: Diffuse intrinsic pontine glioma, H3K27M-wild type, and WHO grade II. ${ }^{18} \mathrm{~F}-\mathrm{DOPA}$ PET shows absence of tracer uptake in the lesion (T/N: 0.95; T/S: 0.45). ADC and ASL images demonstrate increased diffusion (rADC min: 1.26) and low perfusion (rCBF max: 0.78). CE T1-weighted MR image does not show contrast enhancement. ${ }^{1} \mathrm{H}-\mathrm{MRS}$ demonstrates normal Cho/NAA (0.93) and Cho/Cr (1.08) peak area ratios. Note: the box on T2-weighted images indicates the region of interest from which the spectra were acquired. Reproduced with permission from Figure 1 of Piccardo et al. [54].

them here as they may prove to be valuable as more data are published. We summarize here those emerging aMRI techniques that are sufficiently developed such that a comparative aMRI with aaPET study in neurooncology has been published. These techniques include chemical exchange saturation transfer (CEST) imaging, MR fingerprinting, and simultaneous single-quantum- and triple-quantum-filtered imaging of ${ }^{23} \mathrm{Na}$ (SISTINA).

CEST is a novel MRI technique in which compounds containing exchangeable protons are selectively saturated and then detected through water signal. Therefore, CEST imaging captures transfer of magnetization from mobile compounds instead of fixed compounds [66]. Amide CEST, also referred to amide proton transfer (APT), uses the amide-bound hydrogen atoms in water to image endogenous proteins. APT is emerging as a tool for cancer and ischemic stroke visualization [67], and its potential for neurooncology imaging is in its early stages of research. Two studies assessing APT CEST compared to aaPET were published during the review period. Schon et al. compared FET PET with APT imaging in 46 newly diagnosed gliomas to assess cellularity and vascularity of the tumor [60]. They found that tumor volume was larger on APT maps than FET PET and larger in GBM than in low-grade glioma. The volume of overlap between APT and FET PET images was high (median Dice score $=0.8555$ ), as both are associated with tumor cellularity (Figure 9).

Park et al. compared MET PET to APT CEST imaging in 43 posttreatment gliomas to assess diagnostic performance [68]. They found a positive correlation between MET uptake and APT in low-grade recurrences $(r=0.47$ and $P<0.001)$ but not high-grade recurrences, where a moderate negative correlation was reported $(r=-0.24$ and $P<0.001)$. For distinguishing recurrence in high-grade gliomas, APT (AUC 0.88 ) performed better than MET PET (AUC 0.71) $(P<0.05)$. These studies highlight the potential for APT CEST in neurooncology, although further research is needed to elucidate its potential compared to the other imaging modalities.

MR fingerprinting is a quantitative MRI mapping technique that measures multiple tissue properties in a single acquisition, by varying MR system settings in a pseudorandom pulse sequence to generate unique signals ("fingerprints") for each of the tissue properties of interest [69]. The demand for radiogenomics in neurooncology is increasing, and so it is important to compare MR fingerprinting with 

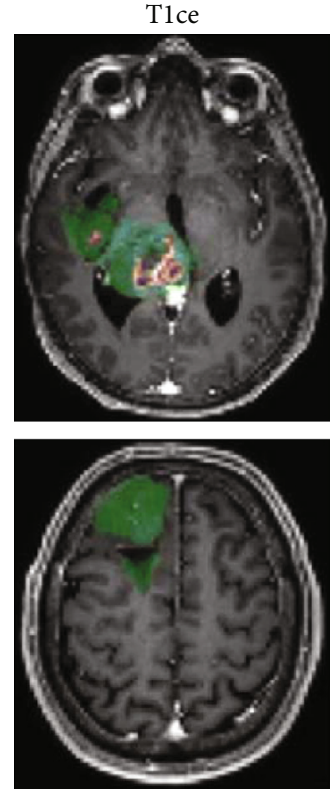

(a)
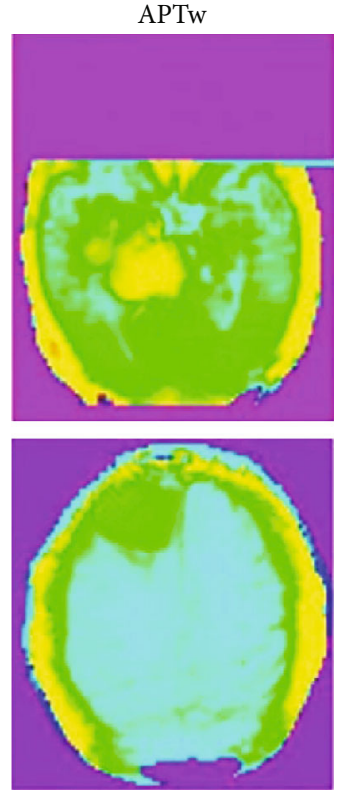

(b)
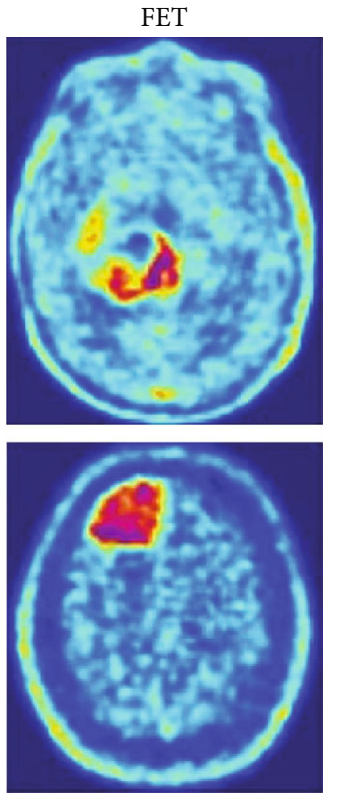

(c)
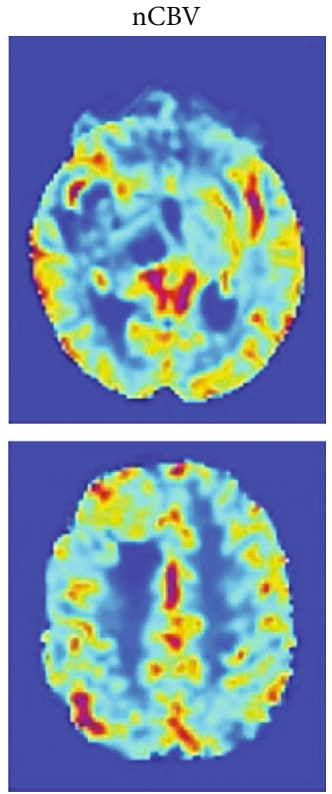

(d)

FIGURE 9: FET PET combined with amide proton transfer (APT) CEST MRI. Example images of a glioblastoma (upper row) and low-grade glioma (lower row). Segmentation (contrast-enhancing tumor (CET) in red, FLAIR hyperintense tumor (FHT) in green) is overlaid on the contrast-enhanced T1-weighted image (a) to show the spatial overlap. FHT in glioblastoma; both amide proton transfer-weighted (APTw) and CBV were significantly $(P<0.001$, respectively) higher in PET-positive areas. Even in low-grade gliomas, a similar trend was observed for the two imaging modalities $(P=0.085$ for APTw and $P=0.045$ for CBV). Reproduced with permission from Figure 2 of Schon et al. [60].

other advanced imaging modalities. Haubold et al. scanned 42 suspected primary brain tumors with FET PET and MR fingerprinting to assess their ability to predict tumor grading and mutational status, relative to histopathology as reference (Figure 10) [70]. They found that these combined modalities were able to differentiate glioma grade (AUC 0.852), ATRX mutation (AUC 0.851), MGMT mutation (AUC 0.757), IDH1 mutation (AUC 0.887), and $1 \mathrm{p} 19 \mathrm{q}$ codeletion (AUC 0.978). These results suggest that this combination of FET PET with MR fingerprinting may prove a clinically valuable tool for noninvasive tumor molecular characterization.

Sodium MRI is a type of imaging that measures sodium concentration in tissue, using the ${ }^{23} \mathrm{Na}$ ion instead of the ${ }^{1} \mathrm{H}$ used in standard MR imaging [71]. The physiochemical limitations of sodium MRI include low MR sensitivity to sodium nucleus, low concentration in the body, and providing only weighted averages of intracellular signals instead of changes in concentration [72]. SISTINA is a specific sequence for sodium MRI that uses a short echo time radial projection with three-pulse triple-quantum preparation, to improve on these limitations [72]. This technique is in early stages of research, but one comparative SISTINA with aaPET study in neurooncology was published during the review period. In a pilot study, Shymanskaya et al. scanned 11 gliomas using FET PET and SISTINA sodium MRI to assess IDH mutational status [73]. The SISTINA parameters were significantly different in IDH-mutated than IDH-wt gliomas, while FET PET parameters were not predictive in this small cohort. Sodium distribution showed no spatial relation to FET uptake. Future studies in larger cohorts, and with a combina- tion of these modalities, will be important for characterizing this potential.

\section{Discussion}

As this review illustrates, additional progress has been made in the past two years on exploring the combination and comparison of aaPET with aMRI across a range of brain tumor populations. Overall, unlike the mixed results seen in the previous older literature, a more consistent theme among most of these studies has emerged whereby aaPET outperforms aMRI for differentiating tumor characteristics such as histologic grade, tumor volume, IDH mutation, and $\mathrm{H} 3 \mathrm{~K} 27 \mathrm{M}$ mutation, and for detecting infiltration, predicting and diagnosing recurrence, and predicting survival. Performance on these targets was almost universally improved by combining imaging modalities, given that they provide complementary rather than duplicative information. This highlights a critical point, that instead of considering aaPET versus aMRI, the future direction of advanced neurooncology imaging should be focused on the combined power of these imaging modalities together.

DWI and PWI emerge from this review as stronger aMRI techniques than MRS, which is limited by data quality issues including limited brain sampling and which is outperformed by MET and FDOPA PET. With regard to spatial correlation of aaPET and PWI, in keeping with previous reports which found mixed results, the new studies are similarly divergent and show inconsistencies in their findings. There is likely a difference in tumor type and/or PWI sequence that is causing 


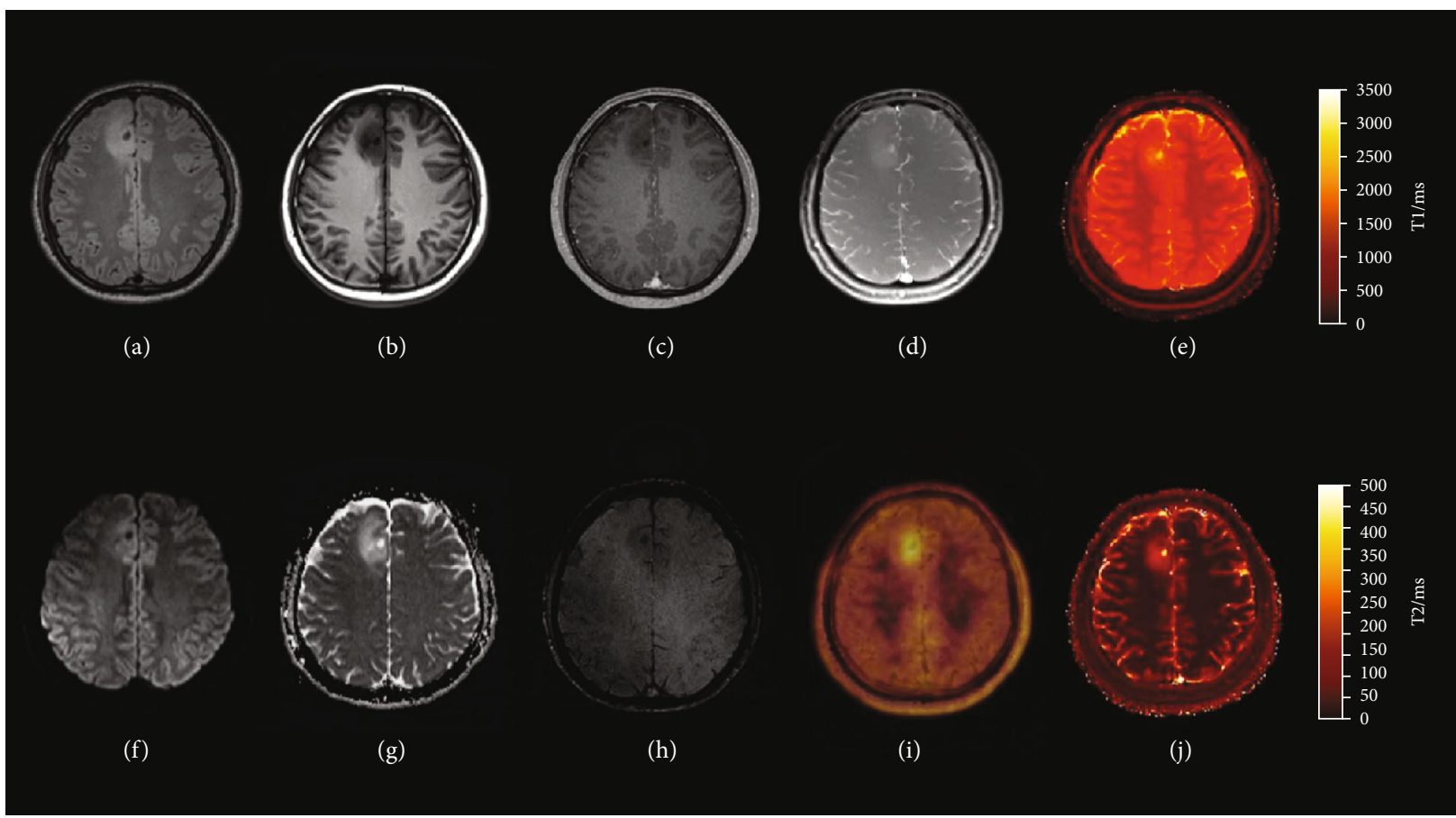

FIGURE 10: FET PET with MRI fingerprinting (MRF). Multiparametric ${ }^{18}$ F-FET PET-MRI and MR fingerprinting of a patient with a lowgrade (WHO II) astrocytoma. (a) 3D FLAIR SPACE (sampling perfection with application optimized contrasts using different flip angle evolution) image. (b) T1-weighted MRI. (c) Postcontrast T1-weighted MRI. (d) MRF M0. (e) MRF T1-weighted image. (f) B1000. (g) ADC. (h) SWI. (i) PET/postcontrast T1-weighted MRI fusion. (j) MRF T2-weighted image. Reproduced with permission from Figure 8 of Haubold et al. [70].

this discrepancy. Nevertheless, it cannot be concluded that these modalities spatially align for all brain tumors. Furthermore, on volumetric analysis, aaPET tumor volume was larger, but after treatment, they tended to equalize in some studies. The previous literature also found that overall aaPET performed better than PWI, which was supported by these recent studies. Overall, both DWI and PWI are valuable imaging techniques for tumor detection, recurrence prediction, recurrence detection, and survival prediction. They were consistently outperformed on these measures by aaPET, including MET, FET, FDOPA, and AMT tracers. It will be worthwhile for researchers and clinicians to consider a dual-modality exam despite the increased overhead, given the superior performance of aaPET vs. aMRI when added to a standard CE-MRI protocol. The future potential for these modalities should be conceptualized in combination, given that they often deliver complementary information.

The emerging aMRI techniques show promise for contributing to neurooncology imaging in the future. MR fingerprinting added meaningfully to the aaPET imaging analysis. APT CEST and sodium MRI outperformed aaPET on some metrics in preliminary studies. It will be interesting to see how the research develops for these techniques, and future efforts should focus on the combinatorial benefit of using these aMRI techniques with aaPET.

4.1. Limitations. The advanced imaging literature reviewed here does suffer from some inherent limitations, such as asynchronous scanning in the majority of the studies, which creates potential issues of misalignment when the scans are subsequently coregistered. Ideally, PET/MRI scanning should be used but, given the limited availability of these scanners at most clinical sites, most centers can minimize time between scans. There is also the difficulty of heterogeneous populations, with studies often targeting different tumors at different time points, leading to results that are not directly comparable. Gliomas are the most represented tumor type in these studies, but attention should also be paid to imaging nonglial tumors and brain metastases. The direct comparison of studies is also complicated by differences in imaging hardware, scanner settings, acquisition protocols, imaging processing software, image processing protocols, and imaging parameter thresholds. Some studies also failed to correlate imaging findings with neuropathology results, which limits their reliability.

\section{Conclusions}

In summary, this review of the recent literature on aaPET with aMRI demonstrates additional encouraging results about the potential for these advanced imaging modalities in neurooncology. Although aaPET outperformed the aMRI techniques in the majority of the studies, there were significant gains when the modalities were combined. Specifically, DWI or PWI when combined with MET, FET, FDOPA, or AMT PET performed well for differentiating tumor grade, tumor volume, select molecular mutations, infiltration, recurrence, and predicting survival. Future efforts should continue to explore the potential for combining aaPET and aMRI in neurooncology imaging, using standardized image 
acquisition and processing protocols and leverage the unique and complementary information gained from each.

\section{Abbreviations}

aaPET: Amino acid positron emission tomography

aMRI: Advanced magnetic resonance imaging

ADC: $\quad$ Apparent diffusion coefficient map

AMT: $\quad{ }^{11} \mathrm{C}$-Alpha-methyl-L-tryptophan

APT: $\quad$ Amide proton transfer

ASL: $\quad$ Arterial spin labeling sequence

AUC: Area under the curve

CE-MRI: Contrast-enhanced magnetic resonance imaging

CEST: Chemical exchange saturation transfer

Cho: Choline

CNI: Choline-to-N-acetylaspartate.

Cr: $\quad$ Creatine

DCE: Dynamic contrast-enhanced sequence

DMG: Diffuse midline glioma

DSC: Dynamic susceptibility contrast sequence

DWI: Diffusion-weighted imaging

FDG: $\quad{ }^{18}$ F-Fluoro-deoxy-glucose

FDOPA: ${ }^{18}$ F-Fluoro-L-dihydroxy-phenylalanine

FET: $\quad{ }^{18}$ F-Fluoroethyl-tyrosine

FLAIR: Fluid-attenuated inversion recovery

GBM: Glioblastoma

IDH: Isocitrate dehydrogenase

MET: $\quad$ L-[Methyl]- ${ }^{11}$ C-methionine

MRI: $\quad$ Magnetic resonance imaging

MRS: $\quad$ Magnetic resonance spectroscopy

NAA: N-acetylaspartate

PET: $\quad$ Positron emission tomography

PWI: $\quad$ Perfusion-weighted imaging

SISTINA: Simultaneous single-quantum- and triplequantum-filtered imaging of ${ }^{23} \mathrm{Na}$

SUV: $\quad$ Standardized uptake value

\section{Data Availability}

The data supporting this systematic review article are from previously reported studies included within the article and which have been cited.

\section{Conflicts of Interest}

The authors declare no potential conflicts of interest with respect to the research, authorship, and/or publication of this article.

\section{Authors' Contributions}

All authors have made substantial contributions to this study and manuscript, as follows: CJ and SM conceived the study idea and provided guidance and oversight to the project. BMS, CJ, and SM made substantial contributions to the study planning. BMS conducted the literature review, data collection and analysis, and manuscript drafting. BMS, CJ, and SM critically reviewed and revised the manuscript for important intellectual content. BMS, CJ, and SM approved the submission of this final version of the manuscript and agree to be accountable for all aspects of the work regarding its accuracy and integrity.

\section{Acknowledgments}

The authors declare the following financial support for the research, authorship, and/or publication of this article: Carilion Clinic and Fralin Biomedical Research Institute at Virginia Tech Carilion School of Medicine.

\section{References}

[1] J. E. Villanueva-Meyer, M. C. Mabray, and S. Cha, "Current clinical brain tumor imaging," Neurosurgery, vol. 81, no. 3, pp. 397-415, 2017.

[2] H. Nandu, P. Y. Wen, and R. Y. Huang, "Imaging in neurooncology," Therapeutic Advances in Neurological Disorders, 2018.

[3] P. Jonsson, A. L. Lin, R. J. Young et al., "Genomic correlates of disease progression and treatment response in prospectively characterized gliomas," Clinical Cancer Research, vol. 25, no. 18, pp. 5537-5547, 2019.

[4] K. O. Almansory and F. Fraioli, "Combined PET/MRI in brain glioma imaging," British Journal of Hospital Medicine, vol. 80, no. 7, pp. 380-386, 2019.

[5] N. L. Albert, M. Weller, B. Suchorska et al., "Response Assessment in Neuro-Oncology Working Group and European Association for Neuro-Oncology recommendations for the clinical use of PET imaging in gliomas," Neuro-Oncology, vol. 18, no. 9, pp. 1199-1208, 2016.

[6] N. Galldiks, K. J. Langen, N. L. Albert et al., "PET imaging in patients with brain metastasis-report of the RANO/PET group," Neuro-Oncology, vol. 21, no. 5, pp. 585-595, 2019.

[7] I. Law, N. L. Albert, J. Arbizu et al., "Joint EANM/EANO/RANO practice guidelines/SNMMI procedure standards for imaging of gliomas using PET with radiolabelled amino acids and [18F]FDG: version 1.0," European Journal of Nuclear Medicine and Molecular Imaging, vol. 46, no. 3, pp. 540-557, 2019.

[8] D. Comar, J. Cartron, M. Maziere, and C. Marazano, "Labelling and metabolism of methionine-methyl-11C," European Journal of Nuclear Medicine, vol. 1, no. 1, pp. 11-14, 1976.

[9] H. J. Wester, M. Herz, W. Weber et al., "Synthesis and radiopharmacology of O-(2-[18F]fluoroethyl)-L-tyrosine for tumor imaging," Journal of Nuclear Medicine, vol. 40, pp. 205-212, 1999.

[10] P. Heiss, S. Mayer, M. Herz, H. J. Wester, M. Schwaiger, and R. Senekowitsch-Schmidtke, "Investigation of transport mechanism and uptake kinetics of O-(2-[18F]fluoroethyl)-L-tyrosine in vitro and in vivo," Journal of Nuclear Medicine, vol. 40, pp. 1367-1373, 1999.

[11] G. Firnau, C. Nahmias, and S. Garnett, "The preparation of (18 F)5-fluoro-DOPA with reactor-produced fluorine-18," The International Journal of Applied Radiation and Isotopes, vol. 24, no. 3, pp. 182-184, 1973.

[12] R. S. Miletich, G. Comi, K. Bankiewicz et al., "6-[18F]FluoroL-dihydroxyphenylalanine metabolism and positron emission tomography after catechol-O-methyltransferase inhibition in normal and hemiparkinsonian monkeys," Brain Research, vol. 626 , no. 1-2, pp. $1-13,1993$. 
[13] C. Juhász, S. Dwivedi, D. O. Kamson, S. K. Michelhaugh, and S. Mittal, "Comparison of amino acid positron emission tomographic radiotracers for molecular imaging of primary and metastatic brain tumors," Molecular Imaging, vol. 13, no. 6, 2014.

[14] M. Diksic, S. Nagahiro, T. L. Sourkes, and Y. L. Yamamoto, "A new method to measure brain serotonin synthesis in vivo. I. Theory and basic data for a biological model," Journal of Cerebral Blood Flow and Metabolism, vol. 10, no. 1, pp. 1-12, 1990.

[15] C. Juhász, D. C. Chugani, O. Muzik et al., "Alpha-methyl-Ltryptophan PET detects epileptogenic cortex in children with intractable epilepsy," Neurology, vol. 60, no. 6, pp. 960-968, 2003.

[16] C. Juhász, D. C. Chugani, O. Muzik et al., "In vivo uptake and metabolism of $\alpha$-[11C]methyl-L-tryptophan in human brain tumors," Journal of Cerebral Blood Flow and Metabolism, vol. 26, no. 3, pp. 345-357, 2005.

[17] F. John, E. Bosnyák, N. L. Robinette et al., "Multimodal imaging-defined subregions in newly diagnosed glioblastoma: impact on overall survival," Neuro-Oncology, vol. 21, no. 2, pp. 264-273, 2019.

[18] D. C. Chugani and O. Muzik, “ $\alpha[$ C-11]Methyl-l-tryptophan PET maps brain serotonin synthesis and kynurenine pathway metabolism," Journal of Cerebral Blood Flow and Metabolism, vol. 20, no. 1, pp. 2-9, 2000.

[19] C. D. Norris, S. E. Quick, J. G. Parker, and N. A. Koontz, "Diffusion MR imaging in the head and neck: principles and applications," Neuroimaging Clinics of North America, vol. 30, no. 3, pp. 261-282, 2020.

[20] C. Ling, F. Shi, J. Zhang, B. Jiang, F. Dong, and Q. Zeng, "In vivo measurement of cytoplasmic organelle water fraction using diffusion-weighted imaging: application in the malignant grading and differential diagnosis of gliomas," Medicine, vol. 98, no. 46, p. e17949, 2019.

[21] J. R. Petrella and J. M. Provenzale, "MR perfusion imaging of the brain: techniques and applications," American Journal of Roentgenology, vol. 175, no. 1, pp. 207-219, 2000.

[22] A. Hilario, A. Hernandez-Lain, J. M. Sepulveda, A. Lagares, A. Perez-Nunez, and A. Ramos, "Perfusion MRI grading diffuse gliomas: impact of permeability parameters on molecular biomarkers and survival," Neurocirugia, vol. 30, no. 1, pp. 11$18,2019$.

[23] K. Gharzeddine, V. Hatzoglou, A. I. Holodny, and R. J. Young, "MR perfusion and MR spectroscopy of brain neoplasms," Radiologic Clinics of North America, vol. 57, no. 6, pp. 11771188, 2019.

[24] A. Lin, T. Tran, S. Bluml, S. Merugumala, H. J. Liao, and B. D. Ross, "Guidelines for acquiring and reporting clinical neurospectroscopy," Seminars in Neurology, vol. 32, no. 4, pp. 432453, 2012.

[25] P. Lohmann, J. M. Werner, N. J. Shah, G. R. Fink, K. J. Langen, and N. Galldiks, "Combined amino acid positron emission tomography and advanced magnetic resonance imaging in glioma patients," Cancers, vol. 11, no. 2, p. 153, 2019.

[26] M. M. D’Souza, R. Sharma, A. Jaimini et al., "11C-MET $\mathrm{PET} / \mathrm{CT}$ and advanced MRI in the evaluation of tumor recurrence in high-grade gliomas," Clinical Nuclear Medicine, vol. 39, no. 9, pp. 791-798, 2014.

[27] F. W. Floeth, D. Pauleit, H. J. Wittsack et al., "Multimodal metabolic imaging of cerebral gliomas: positron emission tomography with [18F]fluoroethyl-L-tyrosine and magnetic resonance spectroscopy," Journal of Neurosurgery, vol. 102, no. 2, pp. 318-327, 2005.

[28] A. Stadlbauer, O. Prante, C. Nimsky et al., "Metabolic imaging of cerebral gliomas: spatial correlation of changes in $\mathrm{O}-(2-18 \mathrm{~F}-$ fluoroethyl)-L-tyrosine PET and proton magnetic resonance spectroscopic imaging," Journal of Nuclear Medicine, vol. 49, no. 5, pp. 721-729, 2008.

[29] J. Mauler, A. A. Maudsley, K. J. Langen et al., "Spatial relationship of glioma volume derived from 18F-FET PET and volumetric MR spectroscopy imaging: a hybrid PET/MRI study," Journal of Nuclear Medicine, vol. 59, no. 4, pp. 603-609, 2018.

[30] C. P. Filss, N. Galldiks, G. Stoffels et al., "Comparison of 18FFET PET and perfusion-weighted MR imaging: a PET/MR imaging hybrid study in patients with brain tumors," Journal of Nuclear Medicine, vol. 55, no. 4, pp. 540-545, 2014.

[31] O. M. Henriksen, V. A. Larsen, A. Muhic et al., "Simultaneous evaluation of brain tumour metabolism, structure and blood volume using [(18)F]-fluoroethyltyrosine (FET) PET/MRI: feasibility, agreement and initial experience," European Journal of Nuclear Medicine and Molecular Imaging, vol. 43, no. 1, pp. 103-112, 2016.

[32] J. Göttler, M. Lukas, A. Kluge et al., "Intra-lesional spatial correlation of static and dynamic FET-PET parameters with MRI-based cerebral blood volume in patients with untreated glioma," European Journal of Nuclear Medicine and Molecular Imaging, vol. 44, no. 3, pp. 392-397, 2017.

[33] A. Verger, C. P. Filss, P. Lohmann et al., "Comparison of 18FFET PET and perfusion-weighted MRI for glioma grading: a hybrid PET/MR study," European Journal of Nuclear Medicine and Molecular Imaging, vol. 44, no. 13, pp. 2257-2265, 2017.

[34] A. Verger, C. P. Filss, P. Lohmann et al., "Comparison of O-(2(18)F-fluoroethyl)-L-tyrosine positron emission tomography and perfusion-weighted magnetic resonance imaging in the diagnosis of patients with progressive and recurrent glioma: a hybrid positron emission tomography/magnetic resonance study," World Neurosurgery, vol. 113, pp. e727-e737, 2018.

[35] A. Jena, S. Taneja, A. Gambhir et al., "Glioma recurrence versus radiation necrosis: single-session multiparametric approach using simultaneous O-(2-18F-fluoroethyl)-L-tyrosine PET/MRI," Clinical Nuclear Medicine, vol. 41, no. 5, pp. e228-e236, 2016.

[36] T. Pyka, D. Hiob, C. Preibisch et al., "Diagnosis of glioma recurrence using multiparametric dynamic 18F-fluoroethyltyrosine PET-MRI," European Journal of Radiology, vol. 103, pp. 32-37, 2018.

[37] V. Dandois, D. Rommel, L. Renard, J. Jamart, and G. Cosnard, "Substitution of 11C-methionine PET by perfusion MRI during the follow-up of treated high-grade gliomas: preliminary results in clinical practice," Journal of Neuroradiology, vol. 37, no. 2, pp. 89-97, 2010.

[38] Y. H. Kim, S. W. Oh, Y. J. Lim et al., "Differentiating radiation necrosis from tumor recurrence in high-grade gliomas: assessing the efficacy of 18F-FDG PET, 11C-methionine PET and perfusion MRI," Clinical Neurology and Neurosurgery., vol. 112, no. 9, pp. 758-765, 2010.

[39] K. R. Roodakker, A. Alhuseinalkhudhur, M. Al-Jaff et al., "Region-by-region analysis of PET, MRI, and histology in en bloc-resected oligodendrogliomas reveals intra-tumoral heterogeneity," European Journal of Nuclear Medicine and Molecular Imaging, vol. 46, no. 3, pp. 569-579, 2019.

[40] F. Cicone, C. P. Filss, G. Minniti et al., "Volumetric assessment of recurrent or progressive gliomas: comparison between F- 
DOPA PET and perfusion-weighted MRI," European Journal of Nuclear Medicine and Molecular Imaging, vol. 42, no. 6, pp. 905-915, 2015.

[41] G. Morana, A. Piccardo, D. Tortora et al., "Grading and outcome prediction of pediatric diffuse astrocytic tumors with diffusion and arterial spin labeling perfusion MRI in comparison with 18F-DOPA PET," European Journal of Nuclear Medicine and Molecular Imaging, vol. 44, no. 12, pp. 2084-2093, 2017.

[42] E. Karavaeva, R. J. Harris, K. Leu et al., "Relationship between [18F]FDOPA PET uptake, apparent diffusion coefficient (ADC), and proliferation rate in recurrent malignant gliomas," Molecular Imaging and Biology, vol. 17, no. 3, pp. 434-442, 2015.

[43] S. Rose, M. Fay, P. Thomas et al., "Correlation of MRI-derived apparent diffusion coefficients in newly diagnosed gliomas with $[18 \mathrm{~F}]$-fluoro-L-dopa PET: what are we really measuring with minimum ADC?," American Journal of Neuroradiology, vol. 34, no. 4, pp. 758-764, 2013.

[44] M. Kinoshita, H. Arita, Y. Okita et al., "Comparison of diffusion tensor imaging and 11C-methionine positron emission tomography for reliable prediction of tumor cell density in gliomas," Journal of Neurosurgery, vol. 125, no. 5, pp. 1136-1142, 2016.

[45] H. Choi, J. C. Paeng, G. J. Cheon et al., "Correlation of 11Cmethionine PET and diffusion-weighted MRI: is there a complementary diagnostic role for gliomas?," Nuclear Medicine Communications, vol. 35, no. 7, pp. 720-726, 2014.

[46] A. Tietze, J. K. Boldsen, K. Mouridsen et al., "Spatial distribution of malignant tissue in gliomas: correlations of $11 \mathrm{C}-\mathrm{L}-$ methionine positron emission tomography and perfusionand diffusion-weighted magnetic resonance imaging," Acta Radiologica, vol. 56, no. 9, pp. 1135-1144, 2015.

[47] I. Popp, S. Bott, M. Mix et al., "Diffusion-weighted MRI and ADC versus FET-PET and GdT1w-MRI for gross tumor volume (GTV) delineation in re-irradiation of recurrent glioblastoma," Radiotherapy and Oncology, vol. 130, pp. 121131, 2019.

[48] A. Castello, M. Riva, B. Fernandes, L. Bello, and E. Lopci, "The role of $11 \mathrm{C}$-methionine $\mathrm{PET}$ in patients with negative diffusion-weighted magnetic resonance imaging: correlation with histology and molecular biomarkers in operated gliomas," Nuclear Medicine Communications, vol. 41, no. 7, pp. 696-705, 2020.

[49] M. Lundemann, P. M. a. Rosenschold, A. Muhic et al., "Feasibility of multi-parametric PET and MRI for prediction of tumour recurrence in patients with glioblastoma," European Journal of Nuclear Medicine and Molecular Imaging, vol. 46, no. 3, pp. 603-613, 2019.

[50] J. M. Werner, G. Stoffels, T. Lichtenstein et al., "Differentiation of treatment-related changes from tumour progression: a direct comparison between dynamic FET PET and ADC values obtained from DWI MRI," European Journal of Nuclear Medicine and Molecular Imaging, vol. 46, no. 9, pp. 18891901, 2019.

[51] J. Lohmeier, G. Bohner, E. Siebert, W. Brenner, B. Hamm, and M. R. Makowski, "Quantitative biparametric analysis of hybrid 18F-FET PET/MR-neuroimaging for differentiation between treatment response and recurrent glioma," Scientific Reports, vol. 9, no. 1, p. 14603, 2019.

[52] N. Verburg, T. Koopman, M. M. Yaqub et al., "Improved detection of diffuse glioma infiltration with imaging combinations: a diagnostic accuracy study," Neuro-Oncology, vol. 22, no. 3, pp. 412-422, 2020.
[53] H. Tatekawa, A. Hagiwara, J. Yao et al., "Voxel-wise and patient-wise correlation of 18F-FDOPA PET, rCBV, and ADC in treatment-naïve diffuse gliomas with different molecular subtypes," Journal of Nuclear Medicine, 2020.

[54] A. Piccardo, D. Tortora, S. Mascelli et al., "Advanced MR imaging and 18F-DOPA PET characteristics of H3K27Mmutant and wild-type pediatric diffuse midline gliomas," European Journal of Nuclear Medicine and Molecular Imaging, vol. 46, no. 8, pp. 1685-1694, 2019.

[55] J.-W. Jeong, C. Juhász, S. Mittal et al., "Multi-modal imaging of tumor cellularity and tryptophan metabolism in human gliomas," Cancer Imaging, vol. 15, no. 1, p. 10, 2015.

[56] T. Beppu, Y. Sato, T. Sasaki et al., "Comparisons between PET with $11 \mathrm{C}$-methyl-L-methionine and arterial spin labeling perfusion imaging in recurrent glioblastomas treated with bevacizumab," Clinical Nuclear Medicine, vol. 44, no. 3, pp. 186-193, 2019.

[57] A. Pala, S. N. Reske, N. Eberhardt et al., "Diagnostic accuracy of intraoperative perfusion-weighted MRI and 5aminolevulinic acid in relation to contrast-enhanced intraoperative MRI and 11C-methionine positron emission tomography in resection of glioblastoma: a prospective study," Neurosurgical Review, vol. 42, no. 2, pp. 471-479, 2019.

[58] Z. Qiao, X. Zhao, K. Wang et al., "Utility of dynamic susceptibility contrast perfusion-weighted MR imaging and 11Cmethionine PET/CT for differentiation of tumor recurrence from radiation injury in patients with high-grade gliomas," American Journal of Neuroradiology, vol. 40, no. 2, pp. 253259, 2019.

[59] G. Dissaux, B. Dissaux, O. E. Kabbaj et al., "Radiotherapy target volume definition in newly diagnosed high grade glioma using 18F-FET PET imaging and multiparametric perfusion MRI: a prospective study (IMAGG)," Radiotherapy and Oncology, vol. 150, pp. 164-171, 2020.

[60] S. Schön, J. Cabello, F. Liesche-Starnecker et al., "Imaging glioma biology: spatial comparison of amino acid PET, amide proton transfer, and perfusion-weighted MRI in newly diagnosed gliomas," European Journal of Nuclear Medicine and Molecular Imaging, vol. 47, no. 6, pp. 1468-1475, 2020.

[61] F. Fraioli, A. Shankar, H. Hyare et al., "The use of multiparametric 18F-fluoro-L-3,4-dihydroxy-phenylalanine PET/MRI in post-therapy assessment of patients with gliomas," Nuclear Medicine Communications, vol. 41, no. 6, pp. 517-525, 2020.

[62] F. John, N. L. Robinette, A. J. Amit-Yousif et al., "Multimodal imaging of nonenhancing glioblastoma regions," Molecular Imaging, vol. 18, 2019.

[63] N. Kudulaiti, T. Qiu, J. Lu et al., "Combination of magnetic resonance spectroscopy and 11C-methionine positron emission tomography for the accurate diagnosis of non-enhancing supratentorial glioma," Korean Journal of Radiology, vol. 20, no. 6, pp. 967-975, 2019.

[64] S. Kebir, L. Lazaridis, M. Weber et al., "Comparison of L-methyl-11C-methionine PET with magnetic resonance spectroscopy in detecting newly diagnosed glioma," Clinical Nuclear Medicine, vol. 44, no. 6, pp. e375-e381, 2019.

[65] M. Goryawala, E. Saraf-Lavi, N. Nagornaya, D. Heros, R. Komotar, and A. A. Maudsley, "The association between whole-brain MR spectroscopy and IDH mutation status in gliomas," Journal of Neuroimaging, vol. 30, pp. 58-64, 2019.

[66] P. C. van Zijl and N. N. Yadav, "Chemical exchange saturation transfer (CEST): what is in a name and what isn't?," Magnetic Resonance in Medicine, vol. 65, no. 4, pp. 927-948, 2011. 
[67] B. Wu, G. Warnock, M. Zaiss et al., "An overview of CEST MRI for non-MR physicists," EJNMMI Physics, vol. 3, no. 1, p. 19, 2016.

[68] J. E. Park, J. Y. Lee, H. S. Kim et al., “Amide proton transfer imaging seems to provide higher diagnostic performance in post-treatment high-grade gliomas than methionine positron emission tomography," European Radiology, vol. 28, no. 8, pp. 3285-3295, 2018.

[69] A. Panda, B. B. Mehta, S. Coppo et al., "Magnetic resonance fingerprinting-an overview," Current Opinion in Biomedical Engineering, vol. 3, pp. 56-66, 2017.

[70] J. Haubold, A. Demircioglu, M. Gratz et al., "Non-invasive tumor decoding and phenotyping of cerebral gliomas utilizing multiparametric 18F-FET PET-MRI and MR fingerprinting," European Journal of Nuclear Medicine and Molecular Imaging, vol. 47, no. 6, pp. 1435-1445, 2020.

[71] G. Madelin, J. S. Lee, R. R. Regatte, and A. Jerschow, "Sodium MRI: methods and applications," Progress in Nuclear Magnetic Resonance Spectroscopy, vol. 79, pp. 14-47, 2014.

[72] D. P. Fiege, S. Romanzetti, C. C. Mirkes, D. Brenner, and N. J. Shah, "Simultaneous single-quantum and triple-quantumfiltered MRI of 23Na (SISTINA)," Magnetic Resonance in Medicine, vol. 69, no. 6, pp. 1691-1696, 2013.

[73] A. Shymanskaya, W. A. Worthoff, G. Stoffels et al., "Comparison of [18F] fluoroethyltyrosine PET and sodium MRI in cerebral gliomas: a pilot study," Molecular Imaging and Biology, vol. 22, no. 1, pp. 198-207, 2020. 